\title{
Nanogenerators for Self-Powered Gas Sensing
}

\author{
Zhen Wen ${ }^{1} \cdot$ Qingqing Shen ${ }^{1} \cdot$ Xuhui Sun ${ }^{1}$
}

Received: 2 February 2017 / Accepted: 30 March 2017/Published online: 6 May 2017

(C) The Author(s) 2017. This article is an open access publication

\begin{abstract}
Looking toward world technology trends over the next few decades, self-powered sensing networks are a key field of technological and economic driver for global industries. Since 2006, Zhong Lin Wang's group has proposed a novel concept of nanogenerators (NGs), including piezoelectric nanogenerator and triboelectric nanogenerator, which could convert a mechanical trigger into an electric output. Considering motion ubiquitously exists in the surrounding environment and for any most common materials used every day, NGs could be inherently served as an energy source for our daily increasing requirements or as one of self-powered environmental sensors. In this regard, by coupling the piezoelectric or triboelectric properties with semiconducting gas sensing characterization, a new research field of self-powered gas sensing has been proposed. Recent works have shown promising concept to realize NG-based self-powered gas sensors that are capable of detecting gas environment without the need of external power sources to activate the gas sensors or to actively generate a readout signal. Compared with conventional sensors, these self-powered gas sensors keep the approximate performance. Meanwhile, these sensors drastically reduce power consumption and additionally reduce the required space for integration, which are significantly suitable for the wearable devices. This paper gives a brief summary about the establishment and latest progress in the fundamental principle, updated progress and potential applications of NG-based self-powered gas sensing system. The development trend in this field is envisaged, and the basic configurations are also introduced.
\end{abstract}

Keywords Nanogenerator $\cdot$ Self-powered $\cdot$ Gas sensing $\cdot$ Piezoelectric $\cdot$ Triboelectric

\section{Introduction}

With the rapid development of internet of things (IoTs) and machine to machine (M2M) technology, the requirement of wireless, sustainable, multi-functional and independent

Xuhui Sun

xhsun@suda.edu.cn

1 Institute of Functional Nano and Soft Materials (FUNSOM), Jiangsu Key Laboratory for Carbon-Based Functional Materials and Devices, and Collaborative Innovation Center of Suzhou Nano Science and Technology, Soochow University, Suzhou 215123, People's Republic of China operation of sensing networks has been becoming increasingly important [1-4]. Considering the large number and small scale of sensors, the implantation of traditional power supply will be a big challenge, and developing selfpowered sensors that can employ the ambient environmental energy and not dependent on a battery or external power source is highly desired [5-7]. These innovative self-powered sensors have been fabricated profiting from the recent advances in environmental energy harvesting technologies, which open an access for developing environmental friendly, independent, remote and mobile, maintenance-free operating nanodevices. The concept of self-powered sensors is based on coupling an external 
energy harvesting or powering unit, such as a solar cell or electromagnet generator, with the functional nanodevice of concern to fulfill the demand for the operation [8, 9].

However, the intermittent and unpredictable nature of solar energy as well as the complexity and heavy weight of electromagnet generator is an inevitable challenge for their expansion as a reliable power supply system to some extent. Since 2006, Zhong Lin Wang's group has firstly fabricated a novel piezoelectric nanogenerator (PENG) that could convert environmental mechanical energy into electrical energy by means of piezoelectric zinc oxide nanowires (NWs) [10]. After that, in early 2012, they invented a triboelectric nanogenerator (TENG) based on the conjunction of triboelectrification and electrostatic induction with higher output and more available materials [11-13]. Most recently, Wang has presented the fundamental theory of the NGs starting from the Maxwell equations. In the Maxwell's displacement current, the second term $\partial \mathrm{P} / \partial \mathrm{t}$ in the Maxwell's displacement current is directly related to the output electric current of the nanogenerators (NGs), meaning that NGs are the applications of Maxwell's displacement current in energy and sensors [14].

As new power generation technologies, NGs can be used to convert mechanical energy into electricity; on the other hand, by analyzing the electrical output signals (including $V_{\mathrm{oc}}, J_{\mathrm{sc}}$, frequency, etc.), information on the mechanical input (magnitude and frequency) can be successfully retrieved [15-19]. Since this sensing technology originates from the output signals of the NG itself, no external power source is required to apply onto the device, which is a unique advantage over conventional sensing technologies. Since then, by correlating the mechanical input with many other parameters, various self-powered prototypes have been realized for different practical applications, mainly including two aspects, one is physical sensing system, such as pressure detection [20, 21], motion sensing [22, 23], acoustic sensing [24, 25], security check [26, 27], medical science [28, 29], and even implantable biosensors [30, 31], and another is chemical sensing system, such as water splitting [32, 33], cleaning pollution [34, 35], anti-corrosion protection [36, 37], electrochromic reaction [38, 39], and electrochemical active sensor [40, 41]. Among abovementioned applications, monitoring the concentration of gases is one of the most important permanent requirements in many fields of industrial processes as well as in everyday life. Applications typically involve safety supervision of important process parameters, environmental monitoring and issues concerning the air quality [42-45]. Owing to the high demand for reliable, independent, minimization, sustainable, maintenance-free and continuous operation of gas sensing devices, one major field is the improvement in selfpowered system. The self-powered gas sensing system based on NGs will be an optimum solution.
In reviewing the studies, the recent works have shown promising concept to realize self-powered gas sensors that are capable of detecting gases using NGs as external power sources to actively generate a readout signal or to activate the sensor-gas interaction. These self-powered gas sensors drastically reduce power consumption compared with conventional sensors and additionally reduce the required space for integration. Herein, the objective of this paper focuses on a review of the fundamental principle, updated progress and potential applications of NG-based selfpowered gas sensing system. The development trend in this field is envisaged, and the basic configurations are also introduced.

\section{PENG-Based Self-powered Gas Sensing System}

The concept of a PENG was firstly presented for converting nanoscale mechanical energy into electricity in 2006, by scanning across vertical piezoelectric $\mathrm{ZnO} \mathrm{NW}$ with a conductive atomic force microscope (AFM) tip, as shown in Fig. 1 [10]. The fundamental of electricity generation in piezoelectric materials is the breaking of central symmetry in the crystal structure under external force, thereby forming a piezoelectric potential, or piezopotential. When a piezoelectric NW is deflected, a piezoelectric potential can be generated on the side surfaces due to the crystal lattice distortion. For $\mathrm{ZnO} \mathrm{NWs}$, the tensile side surface gives a positive potential, while a negative potential appears on the compressive side surface [47, 48]. In 2012, Seong Min Kim and co-workers theoretically investigated that external surface charges on ZnO NWs or AlN nanotubes (NTs) can affect the piezoelectric behavior under uniform compression. The free-carrier depletion caused by negative surface charges via surface functionalization on vertically compressed $\mathrm{ZnO}$ and AlN NWs/NTs indicates the enhancement of piezoelectric potential is due to the free carriers being fully depleted at the critical surface charge density $[49,50]$. Since then, substantial progress has been made to couple the piezoelectric and functional properties, aiming at achieving PENG-based self-powered sensing system $[6,51]$. On the basis of the piezoelectric-semiconductor materials such as $\mathrm{ZnO}, \mathrm{GaN}$ and $\mathrm{CdS}$ with wurtzite or zincblende structure, the emerging field of PENG-based selfpowered sensing system has been demonstrated in different forms, such as self-powered UV sensor [52] and selfpowered $\mathrm{pH}$ sensor [53].

Recently, a new research field mainly aimed at constructing a portable gas sensing system without using external electric power. By coupling the piezoelectric and gas sensing characteristics, the piezoelectric output of PENG could act as both the power source and sensing signal. The gas adsorption on the surface of piezoelectric- 


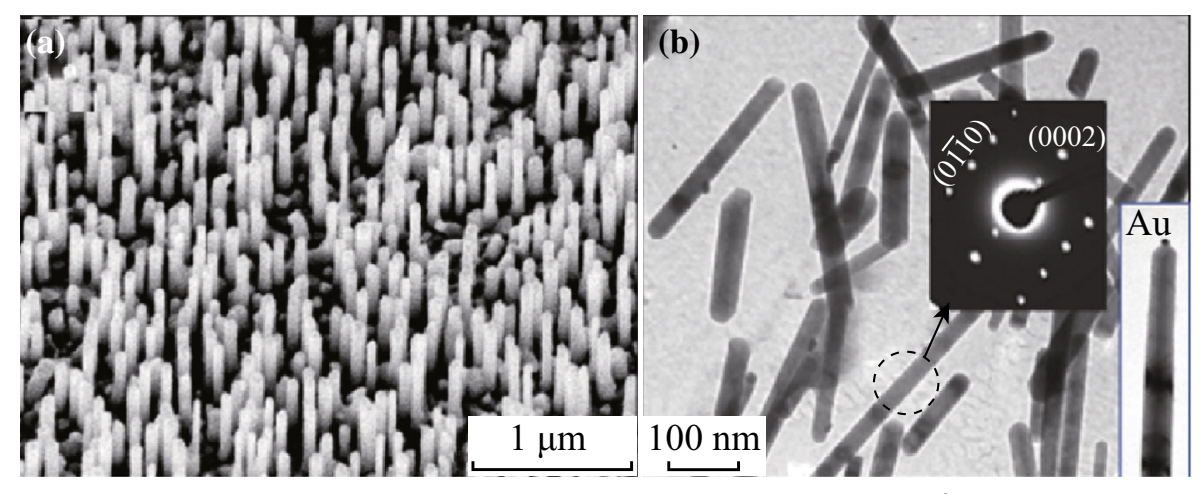

(c)
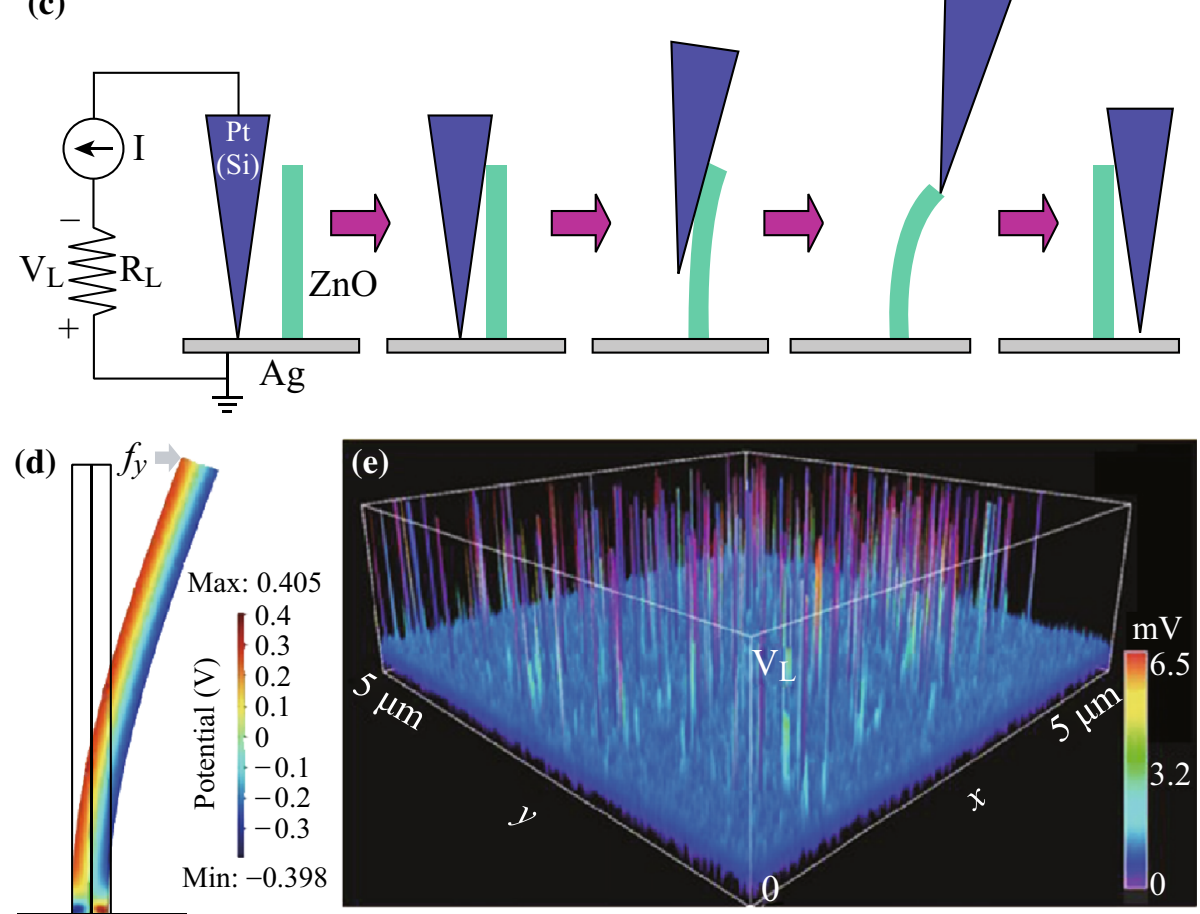

Fig. 1 Design for converting nanoscale mechanical energy into electrical energy by a vertical piezoelectric ZnO NW-based PENG. a SEM images of aligned $\mathrm{ZnO} N W s$ grown on $\alpha-\mathrm{Al}_{2} \mathrm{O}_{3}$ substrate. b TEM images of $\mathrm{ZnO}$ NWs, showing the typical structure of the NW without an Au particle or with a small Au particle at the top (inset at center shows SAED pattern). c Experimental setup and procedures for generating electricity by deforming a piezoelectric NW with a conductive AFM tip. d Potential distribution for a $\mathrm{ZnO} \mathrm{NW}$ in side view. e Electric signal collected by AFM on a $\mathrm{ZnO} \mathrm{NW}$ array. Figures adapted from $[10,46]$

semiconductor nanomaterials can change the free-carrier density, which can vary the piezoelectric output upon applied deformation through the piezoelectric screening effect. The key to realize the self-powered sensor is to choose the appropriate piezo-gas sensing materials. In this section, we would like to introduce the PENG-based selfpowered gas sensing system systematically in the form of nanomaterials.

\subsection{Pure ZnO Nanomaterials}

$\mathrm{ZnO}$, benefiting from lacking central symmetry in wurtzite structures, exhibits strong piezoelectric properties in addition to having a large exciton binding energy and an excellent gas sensing property [54, 55]. Thus, it has potential for application in diverse fields, particularly in realizing a new generation of self-powered sensing system [56-58].

In this regard, Xinyu Xue and co-workers, for the first time, reported the application of an unpackaged PENG based on $\mathrm{ZnO} N W s$ as a self-powered active $\mathrm{H}_{2} \mathrm{~S}$ gas sensor, as shown in Fig. 2. This device is composed of three major components: Ti foil acts as both the substrate for the $\mathrm{ZnO} \mathrm{NW}$ arrays and the conductive electrode that collects the piezoelectric voltage signal generated by $\mathrm{ZnO}$ NWs when being deformed by external compression. As the counter-electrode, a sheet of flexible $\mathrm{Al}$ foil is positioned on top of the $\mathrm{ZnO} \mathrm{NW}$ arrays. In order to ensure 


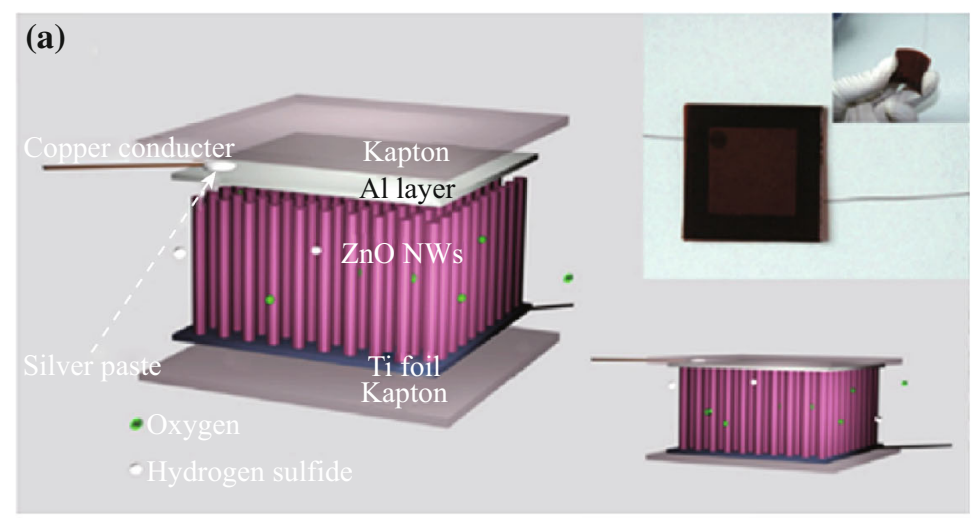

(d)
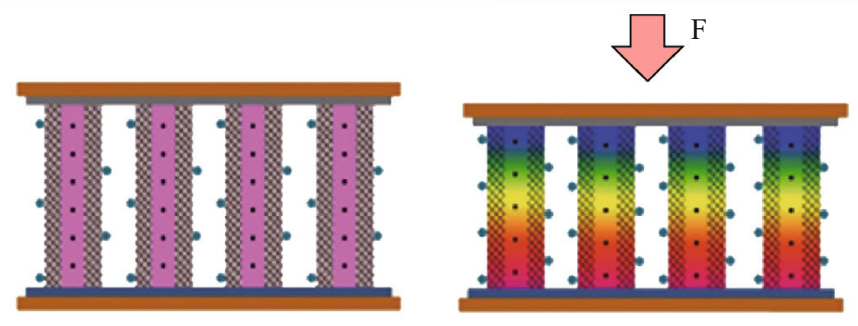

- Kapton $\quad$ Al layer $=\mathrm{ZnO}$ NWs Depletion layer $\quad$ Ti foil - Oxygen · Electron
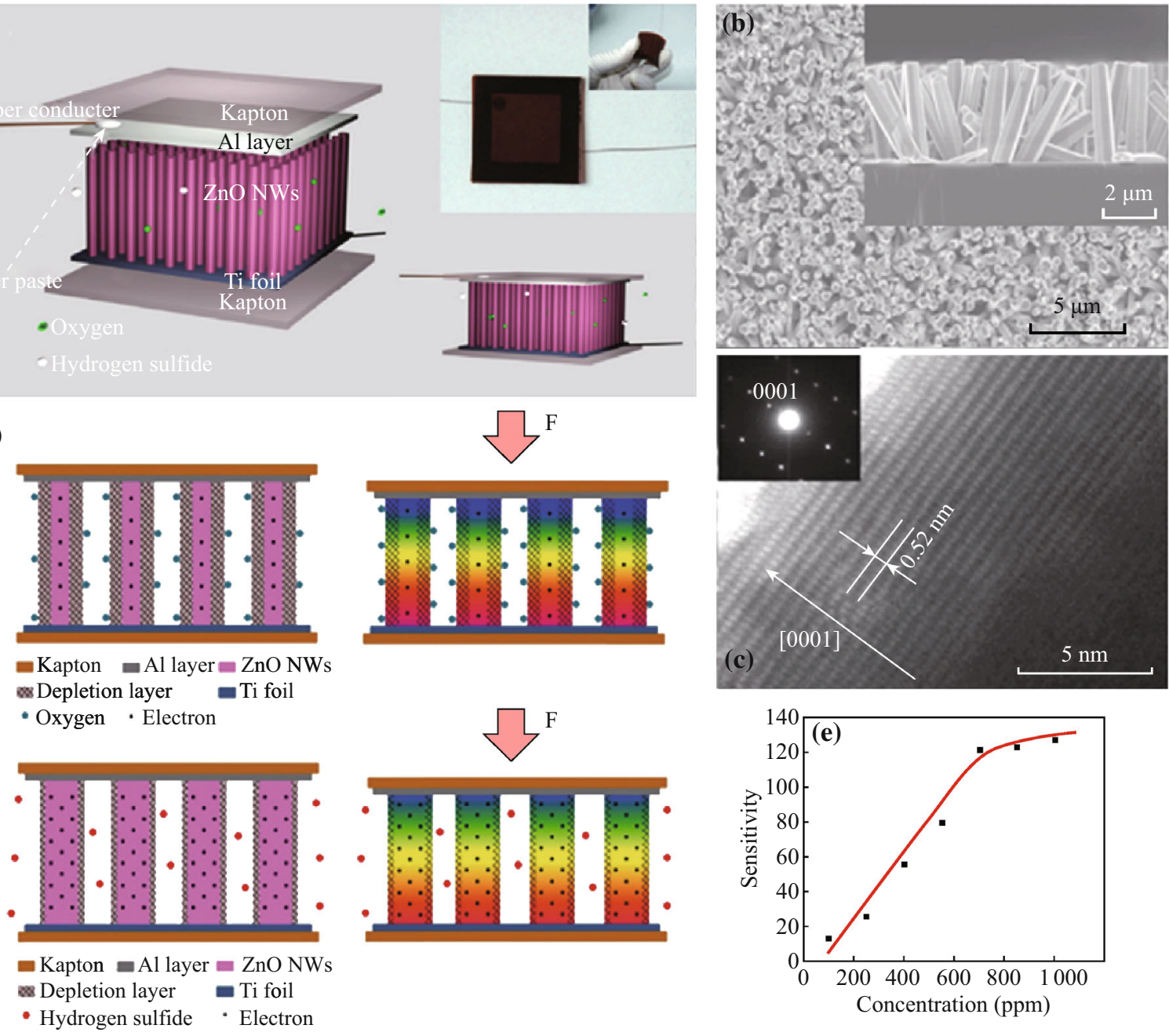

Fig. 2 Surface free-carrier screening effect on the output of a ZnO NW-based PENG and its potential as a self-powered active gas sensor. a Schematic diagram showing the structural design of the PENG (the inset is a photograph of a typical device). b SEM image of ZnO NW arrays grown on Ti foil in a top view (the inset shows SEM the cross-sectional view image). c HRTEM and SAED pattern taken from the tip region of a $\mathrm{ZnO}$ NW. d The working mechanism of the PENG was driven by compressive strain. $\mathrm{e}$ The dependence of sensitivity on the ranging concentrations of $\mathrm{H}_{2} \mathrm{~S}$ vapor. Figures adapted from [59]

good contact, the device was beat repeatedly for more than 40 min by a vibration shaker. Two sheets of Kapton board are fixed at two sides as bracing frames (Fig. 2a). Scanning electron microscope (SEM) images of top view and crosssectional view of the $\mathrm{ZnO} \mathrm{NW}$ arrays, respectively, reveal their diameters of $500 \mathrm{~nm}$ and lengths of $\sim 5 \mu \mathrm{m}$ (Fig. $2 \mathrm{~b}$ ). A high-resolution transmission electron microscope (HRTEM) image and the corresponding selected area electron diffraction (SAED) pattern taken from the tip region of a $\mathrm{ZnO} \mathrm{NW}$ indicate that the $\mathrm{ZnO} \mathrm{NW}$ is single crystalline with a length direction along the $c$-axis (Fig. 2c). As the $c$-axis of $\mathrm{ZnO} \mathrm{NW}$ is under externally applied deformation, a piezoelectric field is created along the surface. The output of a PENG fabricated using $\mathrm{ZnO}$ NW arrays is largely influenced by the density of the surface charge carriers at the NW surfaces. Adsorption of gas molecules could modify the surface carrier density through a screening effect; thus, the output of the PENG is sensitive to the gas concentration (Fig. 2d). The demonstrated sensitivity to $\mathrm{H}_{2} \mathrm{~S}$ to a level is as low as $100 \mathrm{ppm}$. The sensitivity against 100, 250, 400, 550, 700, 850 and $1000 \mathrm{ppm} \mathrm{H}_{2} \mathrm{~S}$ is about 13.1, 25.5, 55.7, 79.3, 121.7, 122.8 and $127.3 \%$, respectively (Fig. 2e). It is the first work which demonstrates the piezoelectric signal generated by pure $\mathrm{ZnO}$ NWs acts not only as a power source, but also as a response signal to the gas, suggesting a possible approach as a self-powered gas sensor [59]. The piezoelectric and gas sensing properties of $\mathrm{ZnO} \mathrm{NWs}$ are coupled into one single physical process through the atmosphere-dependent screen effect on the piezoelectric output. This new 
mechanism opens a new direction for the development of the next generation of gas sensors and expands the scope of self-powered nanosystems.

Followed work of 2D ZnO nanosheets (NSs) network for a room-temperature self-powered humidity sensor has also been demonstrated [60]. However, compared with the traditional metal oxide semiconductor-based gas sensors, it is highly expected to achieve high response and selectivity of self-powered active gas sensor. Much efforts have been made to improve the sensing performance, including doping noble metal, introducing heterostructures, decorating proper element and UV enhancement [61-63].

\subsection{Noble Metal-Doped ZnO Nanomaterials}

In gas sensing field, the conductivity response is determined by the efficiency of catalytic reactions of sensing materials with detected gas taken place at the surface of gas sensing materials. The control of catalytic activity of gas sensor material is one of the most commonly used methods to enhance the performance, especially in detection of low reducing gases, such as ethanol vapor, $\mathrm{H}_{2}$ and $\mathrm{CO}$. Therefore, the pure $\mathrm{ZnO}$ thin film exhibits a very poor sensitivity and selectivity $[64,65]$. Usually, noble metals are high-effective catalysts, which can be used to enhance the reactions on gas sensor surfaces. Thus, if the noble metal decoration can be introduced into self-powered active gas sensing, then higher-performance gas sensor can probably be realized.

In this case, Fu et al. realized a room-temperature selfpowered $\mathrm{H}_{2} \mathrm{~S}$ sensing with high response and selectivity from a $\mathrm{Cu}-\mathrm{ZnO}$ NW-based PENG, as shown in Fig. 3 [66]. The brief fabrication of a $\mathrm{Cu}-\mathrm{ZnO}$-based self-powered/ active $\mathrm{H}_{2} \mathrm{~S}$ sensor was composed of three major parts: $\mathrm{Cu}-$ $\mathrm{ZnO}$ NWs as the power source and the sensing material, Ti and $\mathrm{Al}$ foils as the electrodes and Kapton films as the frames (Fig. 3a). The diameter and length of 5 at $\% \mathrm{Cu}-$ $\mathrm{ZnO}$ NWs are $\sim 230 \mathrm{~nm}$ and $\sim 3.5 \mu \mathrm{m}$, respectively (Fig. 3b). A typical HRTEM image and corresponding SAED pattern show that the $\mathrm{Cu}-\mathrm{ZnO} \mathrm{NW}$ grows along the [0001] direction, and no secondary growth, visible defects or stacking fault can be observed (Fig. 3c). The enhanced room-temperature $\mathrm{H}_{2} \mathrm{~S}$ sensing performance can be attributed to the coupling of the piezoelectric screening effect of $\mathrm{ZnO} \mathrm{NWs}$ and the synergistic effect of the $\mathrm{Cu}$ dopant. $\mathrm{Cu}$ element has a similar ionic radius and electronic shell structure as $\mathrm{Zn}$. $\mathrm{Cu}$ atoms can replace either substitutional or interstitial $\mathrm{Zn}$ atoms in the $\mathrm{ZnO}$ lattice, and $\mathrm{Cu}$ has higher attraction to $\mathrm{H}_{2} \mathrm{~S}$ than $\mathrm{Zn}$ for the initial bridge-top (H-SH) configuration (Fig. 3d). A continuous respondingrecovering process under compressive force $(30 \mathrm{~N}, 1 \mathrm{~Hz})$

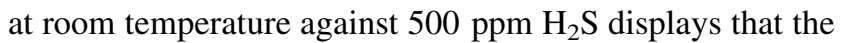
response and recovery time are 100 and $60 \mathrm{~s}$, respectively

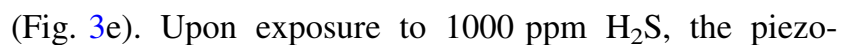
electric output voltage of the device under compressive force decreases from 0.552 to $0.049 \mathrm{~V}$, and the response is up to 1045 , over 8 times larger than that of undoped $\mathrm{ZnO}$ (Fig. 3f).

Such a development of $\mathrm{Cu}-\mathrm{ZnO}$-based self-powered active $\mathrm{H}_{2} \mathrm{~S}$ sensor is an important step for the practical applications in actively detecting gases at room temperature. The catalytic property of $\mathrm{Cu}$ greatly improved the sensing performance. This study demonstrates that introducing elemental doping into the self-powered active gas sensor is a very effective way to enhance its piezo-gas sensing performance. Meanwhile, this study could stimulate research into designing a new series of gas sensors for detecting more gas species at room temperature [66-70].

\subsection{ZnO-Based Heterostructures}

Pure metal oxide materials appear favorable in some functional properties, but very few of them are suitable to all requirements. The heterostructures have been confirmed to improve the performance of metal oxides against various gases. More recent works have reported that upon exposure to the detected gas, the introduction of heterostructures significantly increases or decreases the resistance of the heterostructured nanomaterials, resulting in extremely high sensitivity. Also, the heterostructures can be introduced into self-powered active gas sensors to realize room-temperature gas sensor with high sensitivity.

Nie et al. have fabricated a $\mathrm{CuO} / \mathrm{ZnO}$ heterostructure nanoarray-based self-powered/active gas sensor for roomtemperature $\mathrm{H}_{2} \mathrm{~S}$ detection, as shown in Fig. 4 [71]. The self-powered active gas sensor contains $\mathrm{CuO} / \mathrm{ZnO} \mathrm{PN}$ junction nanoarrays as both piezoelectric and gas sensing materials. Flexible Kapton films as substrates can follow the height profiles of the NW arrays and make effective contacts between the tips of NWs and electrodes (Fig. 4a). The top-view SEM image of $\mathrm{CuO} / \mathrm{ZnO}$ PN-junction nanoarrays indicates that $\mathrm{CuO}$ nanocones are uniformly distributed on the whole surface of $\mathrm{ZnO} \mathrm{NW}$ arrays with the thickness of about $400 \mathrm{~nm}$ (Fig. 4b). Upon exposure to $800 \mathrm{ppm}_{2} \mathrm{~S}$ at room temperature, the piezoelectric output of the device greatly decreases from $0.738 \mathrm{~V}$ (in air) to $0.101 \mathrm{~V}$. The sensitivity increases to 629.8 , much higher than that of bare $\mathrm{ZnO}$ nanoarrays (Fig. 4c). As the device was exposed to $\mathrm{H}_{2} \mathrm{~S}$, the $\mathrm{CuO} / \mathrm{ZnO} \mathrm{PN}$ junction was converted into a $\mathrm{CuS} / \mathrm{ZnO}$ ohmic contact, which greatly increased the electron density in the NW and enhanced the screen effect on the piezoelectric output (Fig. 4d).

This result could stimulate a research trend on designing new composite piezoelectric material for high-performance self-powered active gas sensors. The combination of other components, such as organic composite, were also 
(a)
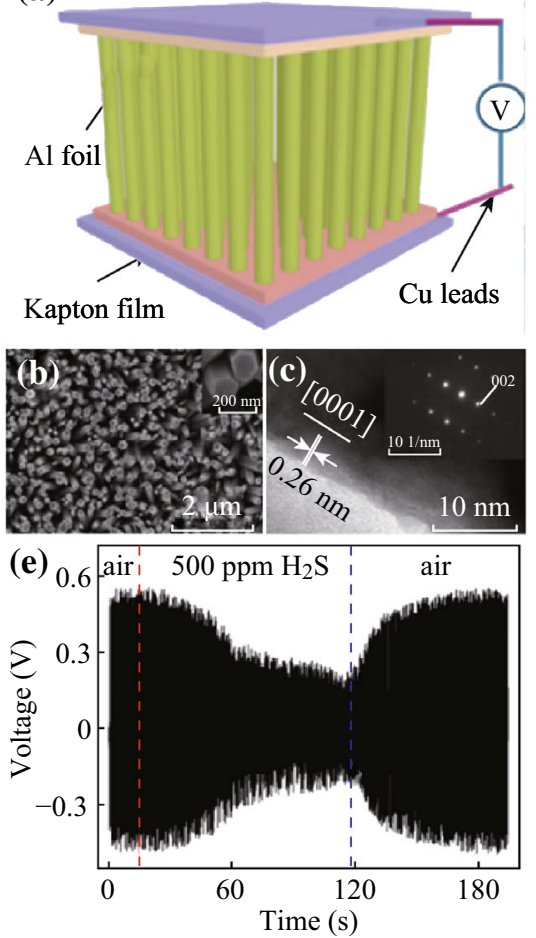

(d)
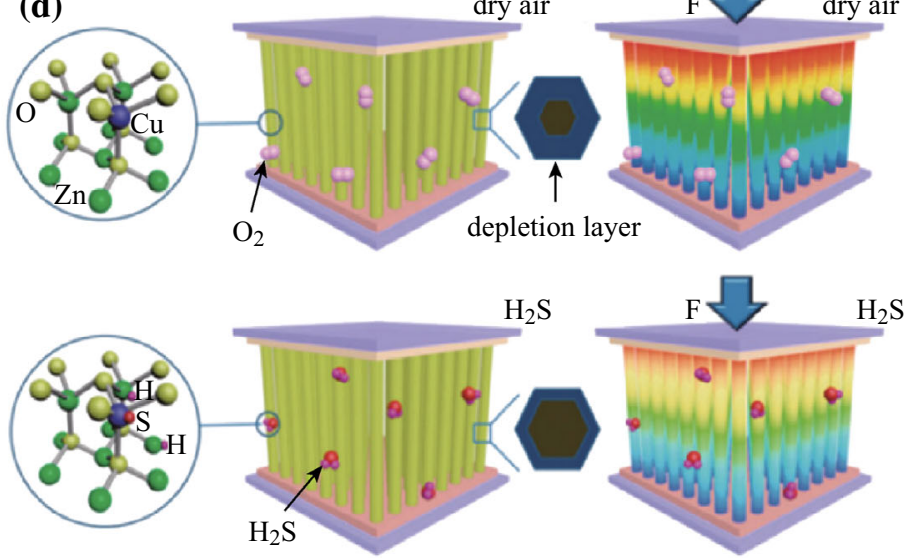

(f)

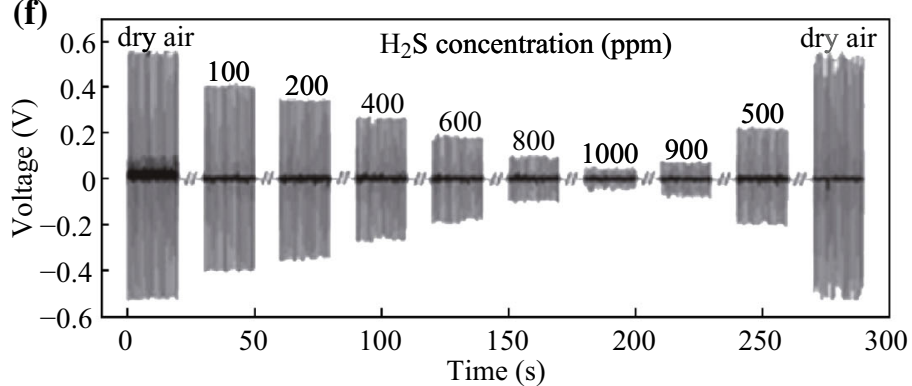

Fig. 3 High response and selectivity of a $\mathrm{Cu}-\mathrm{ZnO}$ NW PENG as a self-powered/active $\mathrm{H}_{2} \mathrm{~S}$ sensor. a Schematic of a $\mathrm{Cu}-\mathrm{ZnO} \mathrm{NW}$ PENG as a self-powered/active $\mathrm{H}_{2} \mathrm{~S}$ sensor. b SEM image of 5 at $\% \mathrm{Cu}-\mathrm{ZnO} N W$ s in top view. c HRTEM image and the SAED pattern of the edge region of a $5 \mathrm{at} \% \mathrm{Cu}-\mathrm{ZnO} \mathrm{NW}$. d Working mechanism of $\mathrm{Cu}-\mathrm{ZnO} \mathrm{NW}$ PENG as a self-powered/active $\mathrm{H}_{2} \mathrm{~S}$ sensor. e A continuous respondingrecovering process of the device against $500 \mathrm{ppm} \mathrm{H}_{2} \mathrm{~S}$. f Piezoelectric output voltage of the device under compressive force at room temperature in dry air and various concentrations of $\mathrm{H}_{2} \mathrm{~S}$. Figures adapted from [66]

investigated. The high feasibility of organic surface modifications in terms of functional groups, as well as their steric and electronic structures might possibly enable the targeted design of various specific gas sensors.

Martin W. G. Hoffmann and co-workers have demonstrated a selective and self-powered gas sensor by microfabricated $p$-Si $/ n-\mathrm{ZnO}$ diodes upon visible-light illumination, as shown in Fig. 5. The selective sensing qualities were introduced by the functionalization of the $n$ $\mathrm{ZnO}$ surface with amine- as well as thiol-terminated organic self-assembled monolayer (SAM), capable of detecting low $\mathrm{NO}_{2}$ concentrations in the ppb range without the need of an external power source (Fig. 5a, b). After patterning of a $p$-Si layer on $\mathrm{SiO}_{2}$ via reactive ion etching, photolithographic methods were used to deposit layers of $20 \mathrm{~nm}$ of ZnO selectively on the $p$-Si sidewalls that served as a seed layer for site selective growth of $n$-ZnO NWs, to form $p$-Si $/ n-\mathrm{ZnO}$ heterojunctions (Fig. $5 \mathrm{c}$ ). Furthermore, the use of an organic SAM facilitated the gas-surface interaction without the need of heat or UV activation, as is required for bare inorganic gas sensors (Fig. 5d). Detailed density functional theory (DFT) simulations of the SAM$\mathrm{NO}_{2}$ binding interactions and subsequent changes of the organic surface group frontier molecular orbitals indicate that the nature of the chemical SAM structure directly determines the gas response of the hybrid material (Fig. 5e). The contrary relative changes of the ionization potential and electron affinity upon $\mathrm{NO}_{2}$ binding for amineand thiol-terminated SAMs correlate well with the experimentally observed sensing results (Fig. 5f).

This work gives an insight into the complex sensing mechanism of inorganic-organic hybrid gas sensors and shows the feasibility of transferring chemical signals from specific organic-gas interactions into active electronic signals solely driven by visible light [72]. Heterostructures can stimulate a research trend on the development of the next generation of room-temperature gas sensors and will further expand the scope for self-powered nanosystems.

\subsection{Non-ZnO Nanomaterials}

It should be noted that $\mathrm{ZnO}$ NWs in the prototype piezodriven active gas sensors may be corroded by $\mathrm{H}_{2} \mathrm{~S}$. Therefore, there is an urgent demand to explore new nanostructured materials that have good gas sensing 

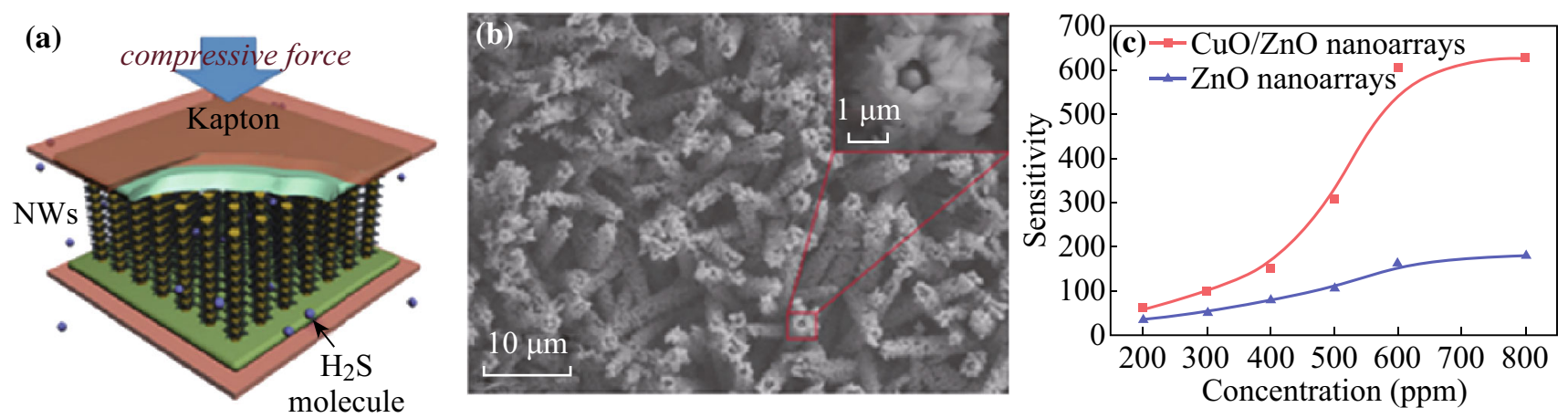

(d)
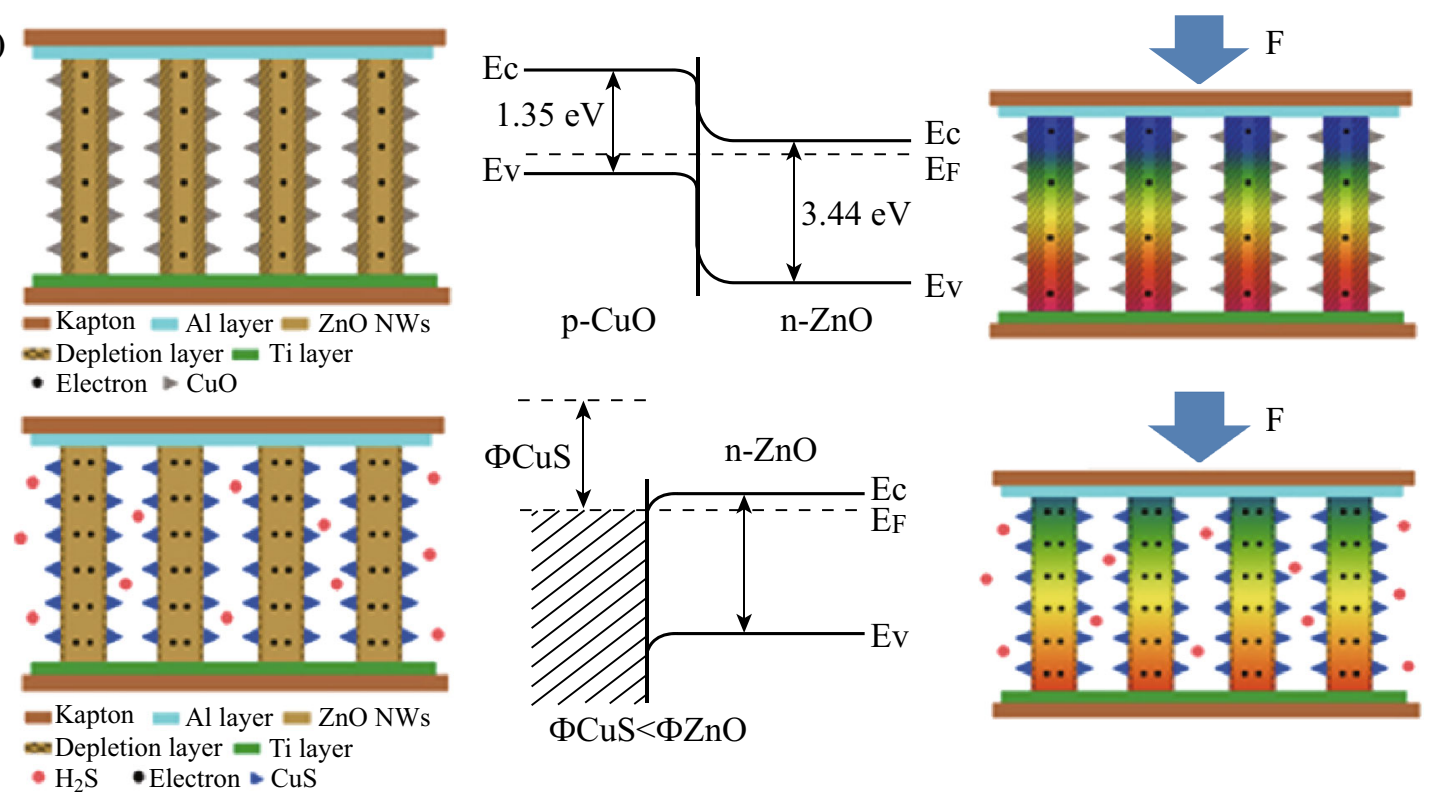

Fig. 4 Conversion of PN junction influencing the piezoelectric output of a $\mathrm{CuO} / \mathrm{ZnO}$ nanoarray PENG and its application as a room-temperature self-powered active $\mathrm{H}_{2} \mathrm{~S}$ sensor. a Schematic diagram showing the structure of the self-powered active gas sensor with two Kapton boards as the frame. b SEM image of $\mathrm{CuO} / \mathrm{ZnO}$ nanoarrays on the top view (the inset is an enlarged view of a selected area). c The sensitivity of the device upon exposure to different concentrations of $\mathrm{H}_{2} \mathrm{~S}$ with one device repeatedly compressed by a constant applied strain of $0.012 \%$ at a frequency of $0.8 \mathrm{~Hz}$. d The $\mathrm{H}_{2} \mathrm{~S}$ sensing mechanism of the self-powered active gas sensor based on $\mathrm{CuO} / \mathrm{ZnO} \mathrm{PN}$-junction nanoarrays driven by compressive strain. Figures adapted from [71]

performance, high piezoelectric output and high chemical stability as well.

By coupling the piezoelectric and gas sensing properties of CdS nanorods (NRs), a flexible piezo-driven self-powered/active $\mathrm{H}_{2} \mathrm{~S}$ sensor has been fabricated by Penglei et al. [73], as shown in Fig. 6. The piezo-driven $\mathrm{H}_{2} \mathrm{~S}$ sensor was fabricated from three parts: CdS NR arrays, Ti foil and Al layer as electrodes, and Kapton boards (Fig. 6a). The CdS nanorods are vertically aligned on the Ti substrate with the average diameter of about $200 \mathrm{~nm}$. The inset of Fig. 6b shows that the cross-sectional shape of the CdS nanorods is hexagonal. The HRTEM image of the tip region of a CdS nanorod and the corresponding SAED pattern reveal that the CdS nanorod is single crystalline with a growth along the [001] direction (Fig. 6c). Upon exposure to $600 \mathrm{ppm}$ $\mathrm{H}_{2} \mathrm{~S}$, the piezoelectric output of the device decreased from
$0.32 \mathrm{~V}$ (in air) to $0.12 \mathrm{~V}$. Such a flexible device can be driven by the tiny mechanical energy in our living environment, such as human finger pinching (Fig. 6d). When the device in $\mathrm{H}_{2} \mathrm{~S}$ is under compressive strain, the screening effect of free electrons is extremely strong because of the high electron density, and the piezoelectric output is lower than that in air (Fig. 6e).

\section{TENG-Based Self-powered Gas Sensing System}

Triboelectrification has been conventionally known since the ancient Greek era and usually taken as a negative effect. However, tactfully based on a conjunction of triboelectrification and electrostatic induction, in 2012, a simple, cost-effective and all-polymer-based flexible 

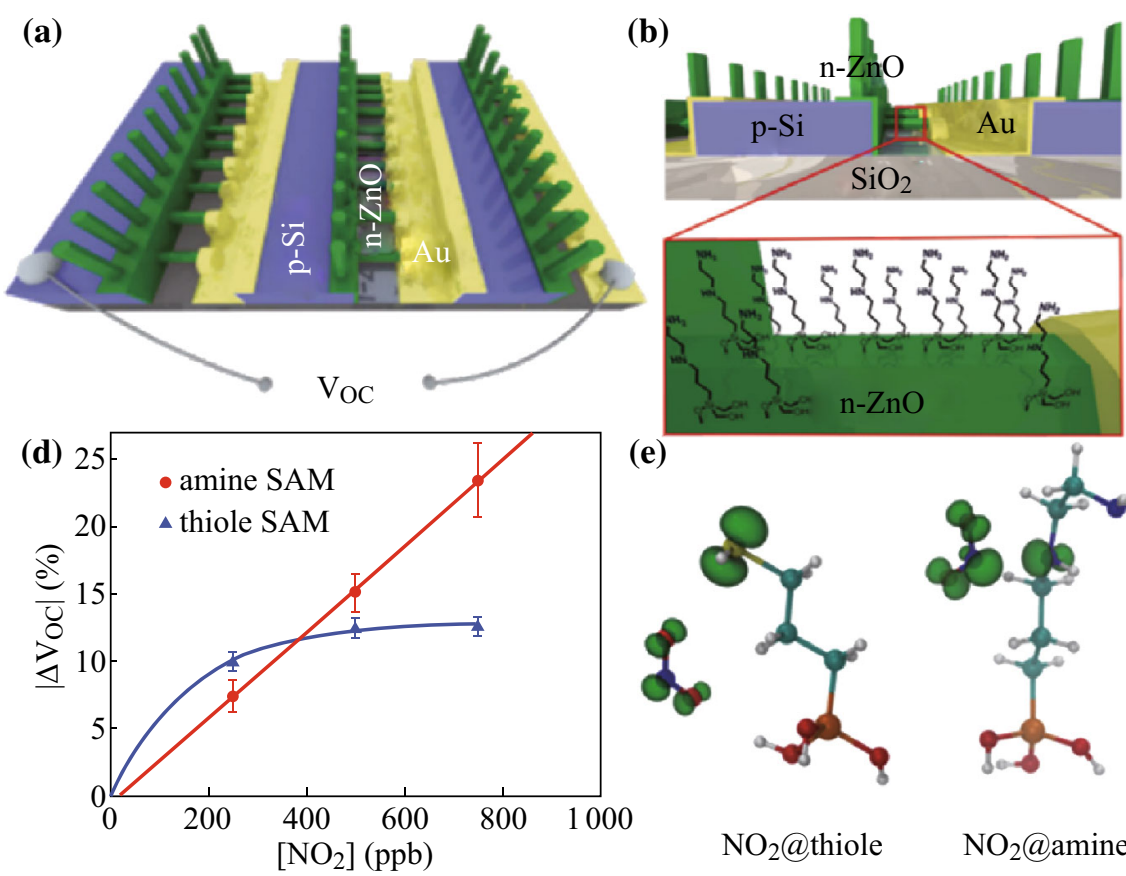

(e)
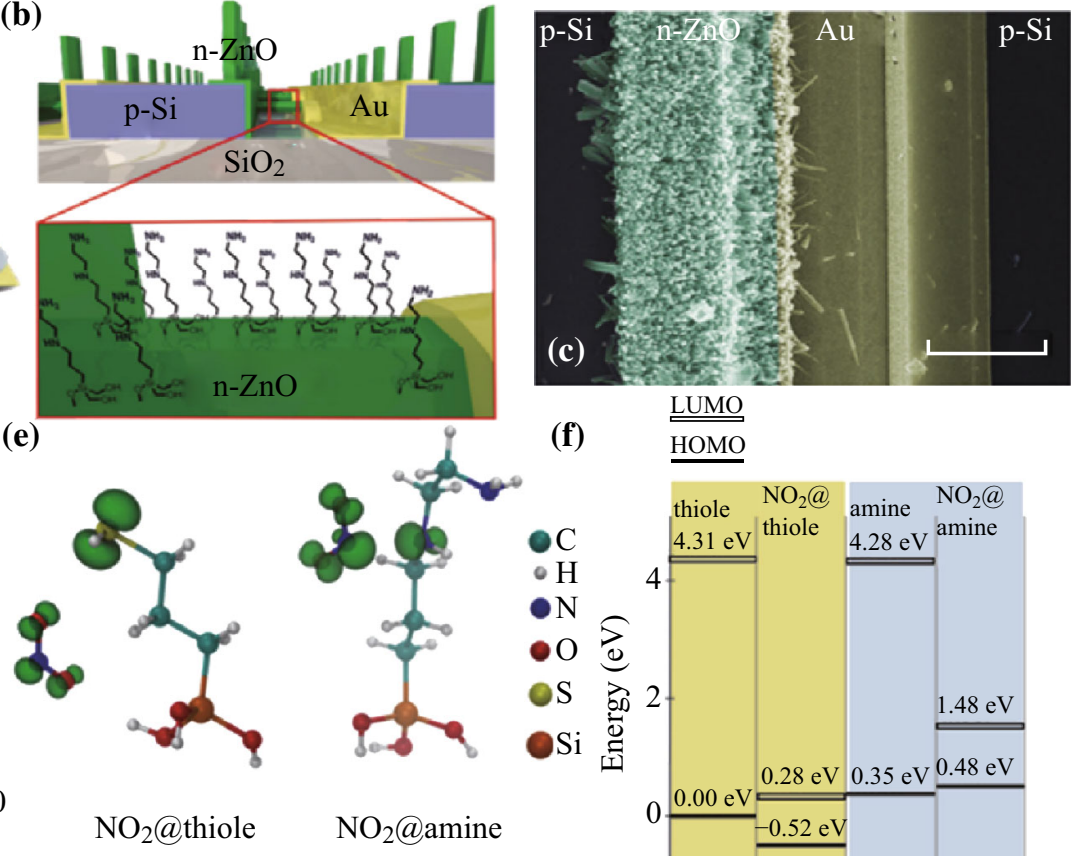

$$
\text { LUMO }
$$

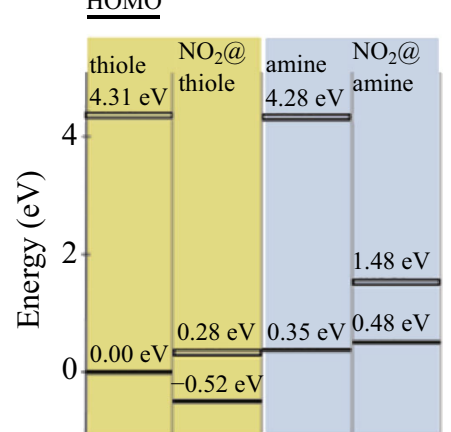

Fig. 5 A highly selective and self-powered gas sensor via organic surface functionalization of $p$-Si/ $n$-ZnO diodes. a Schematic of a singular p-n unit composed of $p$-Si $\mu$-trenches. b The surface of the $n$-ZnO NWs was modified by adding a SAM of functional groups. $\mathbf{c}$ SEM image of a singular $p-\mathrm{Si} / n-\mathrm{ZnO}$ diode (scale bar: $10 \mu \mathrm{m}$ ). d Diagram of the $\Delta V_{\mathrm{oc}}$ response for different $\mathrm{NO}_{2}$ concentrations, for amine- and thiolfunctionalized devices. e Most stable geometries of $\mathrm{NO}_{2}$ molecules adsorbed at the thiol and amine functionalities. $\mathbf{f}$ Energy levels of the $\mathrm{HOMO}$ and LUMO of the functionalities without adsorbed $\mathrm{NO}_{2}$ and with adsorbed $\mathrm{NO}_{2}$. The averaged electrostatic potential from the core region of the $\mathrm{Si}$ atom of each functionality was used as reference potential. The HOMO level of the isolated thiol functionality was set to 0 for convenience. Figures adapted from [72]

TENG was invented by Zhong Lin Wang's group, as shown in Fig. 7 [11, 75]. A TENG mainly consists of two polymer films that have different electron-attracting abilities, with metal films deposited on their back sides as electrodes (Fig. 7a). When the two films contact, friction happens, owing to the natural nanoscale surface roughness, which leads to equal amount but opposite signs of charges generated on the two films' surfaces. Thus, an electric potential is formed at the interface region. When the two films contact and separate, the alternative potential will drive electrons in the external load to flow back and forth (Fig. 7b) [76-81]. Based on such a principle, four different modes of TENGs were invented, vertical contact-separation mode, lateral sliding mode, single-electrode mode and freestanding triboelectric-layer mode, respectively [82-87].

In the basic working principles of TENGs, the amplitudes of generated signals are all proportional to the triboelectric charge density when all of the other conditions are maintained the same [88-90]. The triboelectric charge density is greatly influenced by the surface alteration of certain chemical molecules or environmental factors, so self-powered electrochemical active sensors based on TENGs can be developed [91, 92]. In comparison with PENG, TENG-based gas sensors work simply by the formation of a dipole layer after triboelectric contact and static separation between two materials of the triboelectricseries. The resistivity of the triboelectric material changes due to the chemisorptions of the molecular oxygen species by the surface, which can be affected by a bit of opposite charges [93, 94]. Since 2015, extensive works have been carried out to develop triboelectric-based self-powered active gas sensors, as elaborated in the following.

\subsection{ZnO-Based Triboelectric Materials}

The working mechanism of TENG-based self-powered gas sensors can be ascribed to the coupling of triboelectrification effect and surface reaction of triboelectric materials. Among various triboelectric materials, although $\mathrm{ZnO}$ is not a tribo-series material, it exhibits triboelectric properties due to its finite conductivity characteristics. The discrete surfaces of $\mathrm{ZnO}$ NRs would be desirable for TENG, which require an efficient contact and interface to the surface of triboelectric polymer materials [95]. Regarding combination of outstanding gas sensing property and electrification ability, in 2015, Jeong Min Baik's group proposed the first TENG-based self-powered gas sensor based on triboelectrification by the physical contact between the $\mathrm{ZnO}$ NWs 

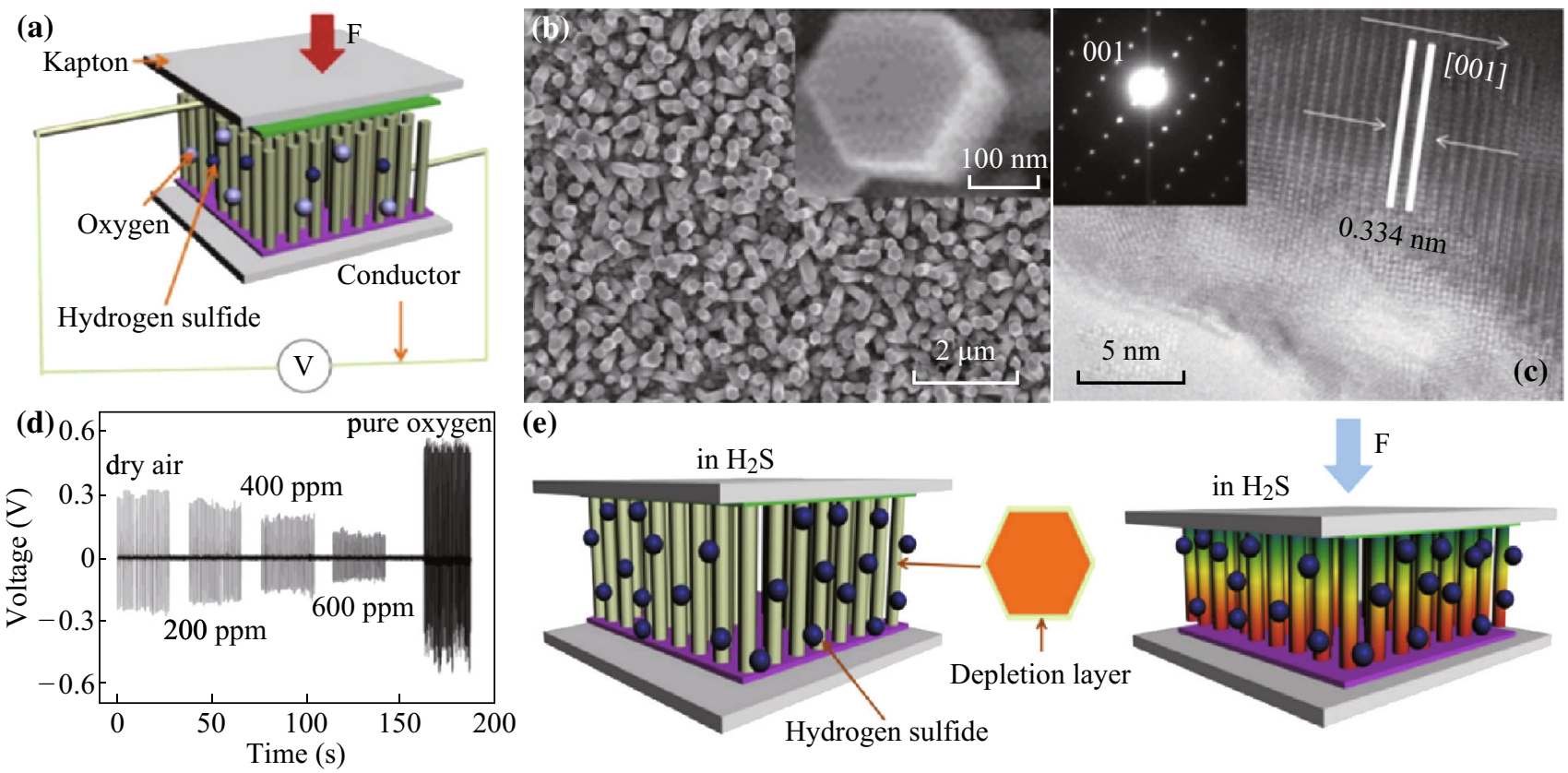

(e)
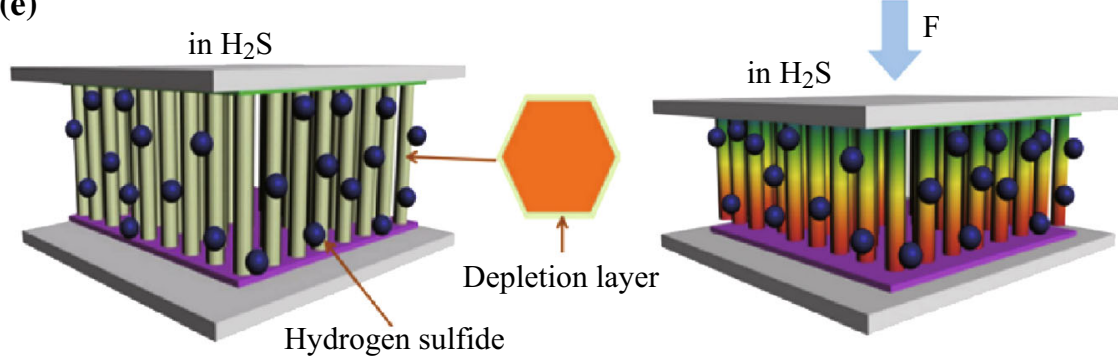

Fig. 6 Synthesis of CdS nanorod arrays and their applications in flexible piezo-driven active $\mathrm{H}_{2} \mathrm{~S}$ sensors. a Schematic diagram showing the structural design of the piezo-driven active $\mathrm{H}_{2} \mathrm{~S}$ sensor based on CdS nanorod arrays. b SEM image of CdS NR arrays grown on Ti foil in a top view (the inset is an enlarged view of a selected area). c HRTEM and SAED pattern of the tip region of a CdS nanorod. $\mathbf{d}$ The piezoelectric voltage response of a device in dry air, $\mathrm{H}_{2} \mathrm{~S}$ and pure oxygen at room temperature. The working mechanism of a CdS piezo-driven active $\mathrm{H}_{2} \mathrm{~S}$ sensor. e The carrier density and depletion layer in CdS nanorods in $\mathrm{H}_{2} \mathrm{~S}$ without compression and piezoelectric output under mechanical deformation. Figures adapted from [73]

and the dielectric layers polytetrafluoroethylene (PTFE) films, and the heterogeneous catalytic reaction occurring on the $\mathrm{ZnO}$ NWs and the decorated $\mathrm{NiO}$ nanoparticles (NPs).

The self-powered gas sensing system has been demonstrated in the form of electronic nose strategy with highly selective gas detection, as shown in Fig. 8. The electronic nose is a two-dimensional microarray where the individual sections orthogonally vary in their properties on account of two response-modifying strategies (Fig. 8a). Along one axis, a NiO NP functionality was applied to the $\mathrm{ZnO} \mathrm{NWs}$ (Fig. 8b). The NiO functionality (Fig. 8c) was found to be more reactive for all volatile organic compound (VOC) gases, whereas only acetone gas was reactive on the surface of the $\mathrm{ZnO} \mathrm{NWs}$ due to its small dissociation energy. The electron transfer to the NWs by the catalytic oxidation increased the triboelectric charge density at both surfaces, thereby increasing the output voltage of the devices. The slow response time also supported the contribution of the catalytic oxidation to the output power. Two dielectric layers [PTFE and polyimide (PI)] with different surface tensions were placed along the orthogonal axis. When the surfaces are exposed to the VOC gases, the output voltage decreases because the molecular species on both surfaces reduce the triboelectrically charged area. The sensor comprising a polyimide layer shows a faster response than the one including a PTFE layer, because of the higher surface energy of PI compared to that of PTFE (Fig. 10d, e). This self-powered sensor may be applicable in many places with limited accessibility to monitor gases and chemicals over long periods of time or in portable applications, such as electronic skins or textiles [96].

In another work, A. S. M. Iftekhar Uddin and GwiySang Chung have successfully fabricated a triboelectricbased $\mathrm{H}_{2}$ sensor (TEHS) by combining a uniformly grown Pd NPs/ZnO NRs/Au/PET (polyester, PET) film with a micropyramid PDMS film. PDMS has already got considerable priority as a structure material due to its greater ability to attract and retain electrons upon contact with any positively charged triboelectric materials (Fig. 9a-c). In air, the TEHS device shows a peak-to-peak $V_{\mathrm{oc}}$ and $I_{\mathrm{sc}}$ of about $5.2 \mathrm{~V}$ and $80 \mathrm{nA}$, respectively. The as-fabricated device also shows an effective and reliable detection ability of $\mathrm{H}_{2}$ molecules at room temperature, in which a continuous decrease in output voltages with increasing $\mathrm{H}_{2}$ gas concentrations was observed (Fig. 9d). A maximum response of $\sim 373 \%$ and a short response time of $100 \mathrm{~s}$ were obtained, while the TEHS was exposed to $10,000 \mathrm{ppm} \mathrm{H}_{2}$ (Fig. 9e). The working mechanism of the TEHS device can be explained through the conjunction of the triboelectric effect and surface reaction mechanism. The polarized charges resulted from the friction (triboelectric effect) also regulated the charge carrier transport 

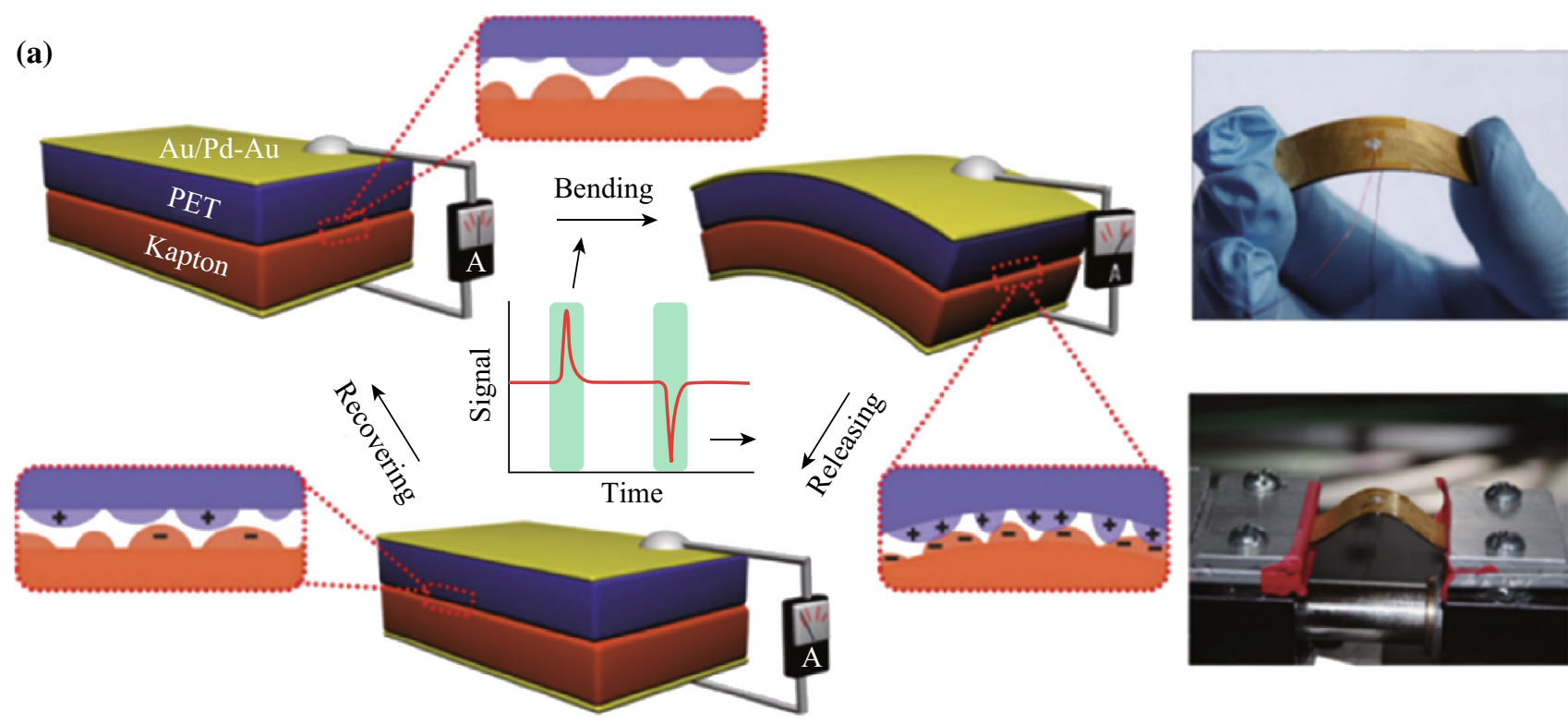

(b)
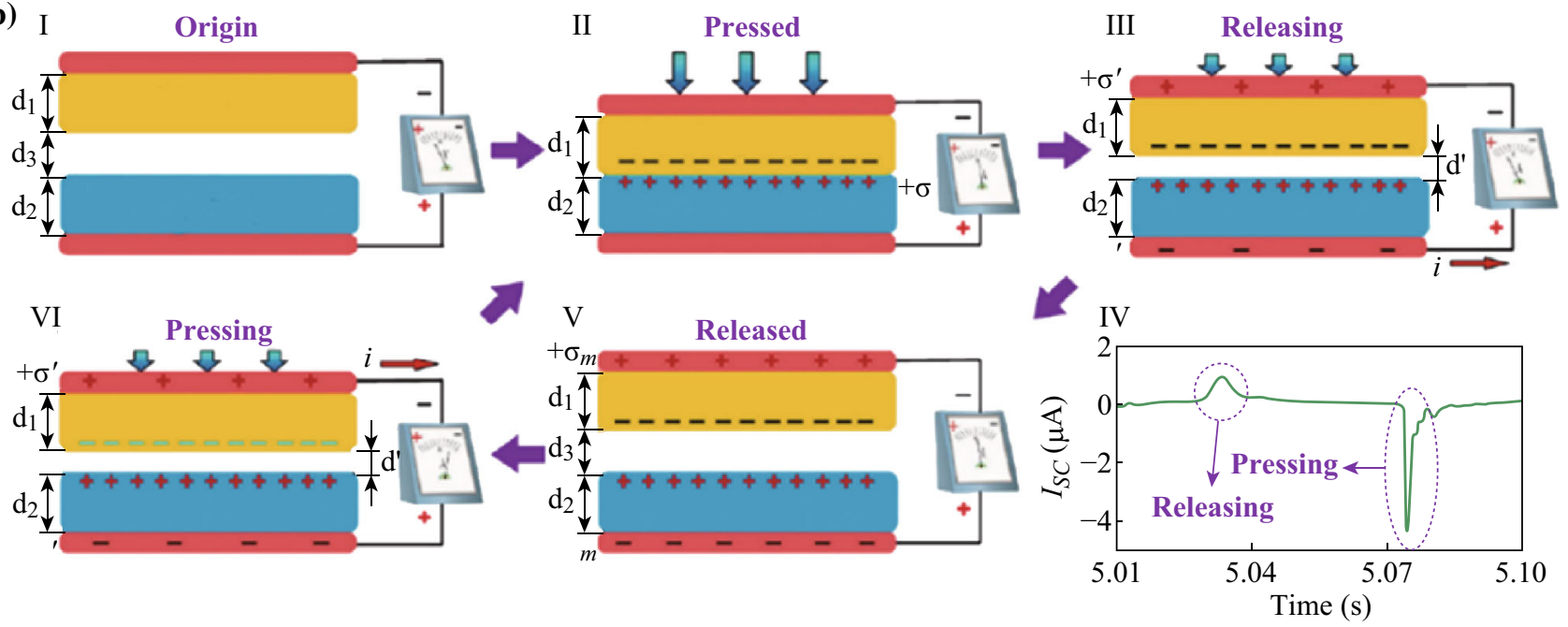

Fig. 7 Invention and working principle of the first TENG that converts mechanical energy into electricity. a The construction and photography images in bending and releasing process and related electrical measurement tests. b Sketches that illustrate the operating principle. Figures adapted from $[11,74]$

through the $\mathrm{Pd} / \mathrm{ZnO}$ interfaces and modulated the interfacial energy at the junction area. Moreover, with the increasing $\mathrm{H}_{2}$ concentrations this phenomenon was accelerated, resulting in further decrease in output voltages (Fig. 9f, g). The aforementioned features of the TEHS device along with the major advantages in gas sensitivity, reliability, cost, scalability, durability and implementation will open up a new paradigm for widespread adoption of self-powered active $\mathrm{H}_{2}$ sensing in the near future.

\subsection{Non-ZnO-Based Triboelectric Materials}

Except for $\mathrm{ZnO}$ nanomaterials, researchers have been looking for other good tribo- as well as gas sensing materials for self-powered gas sensing system, such as conductive polyaniline (PANI), which is a good gas sensing material since PANI chains can react with various volatile organic compounds at room temperature.

Xue et al. fabricated a flexible smelling e-skin that based on the triboelectrification/gas sensing coupling effect of PANI/PTFE/PANI sandwich nanostructures, as shown in Fig. 10. The PTFE film acts as the triboelectrification material. The two PANI films act as both the functional (sensing/triboelectrification) materials and the electrodes (Fig. 10a-c). The flexible e-skin has two operation modes: gas flow (human breath) driving the vibration of PTFE film (Fig. 10d, e); pressure (human motion) driving the movement of PANI films (Fig. 10f, g). The self-powered system 
(a)

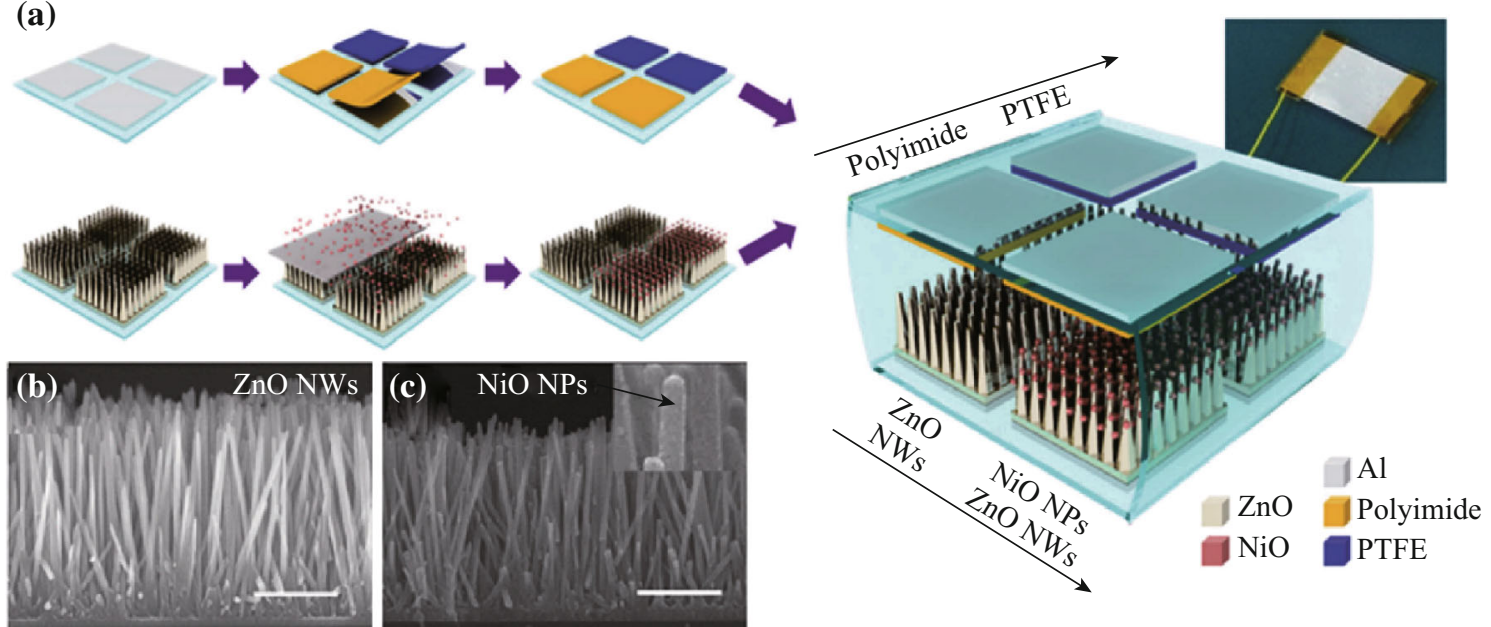

(d)
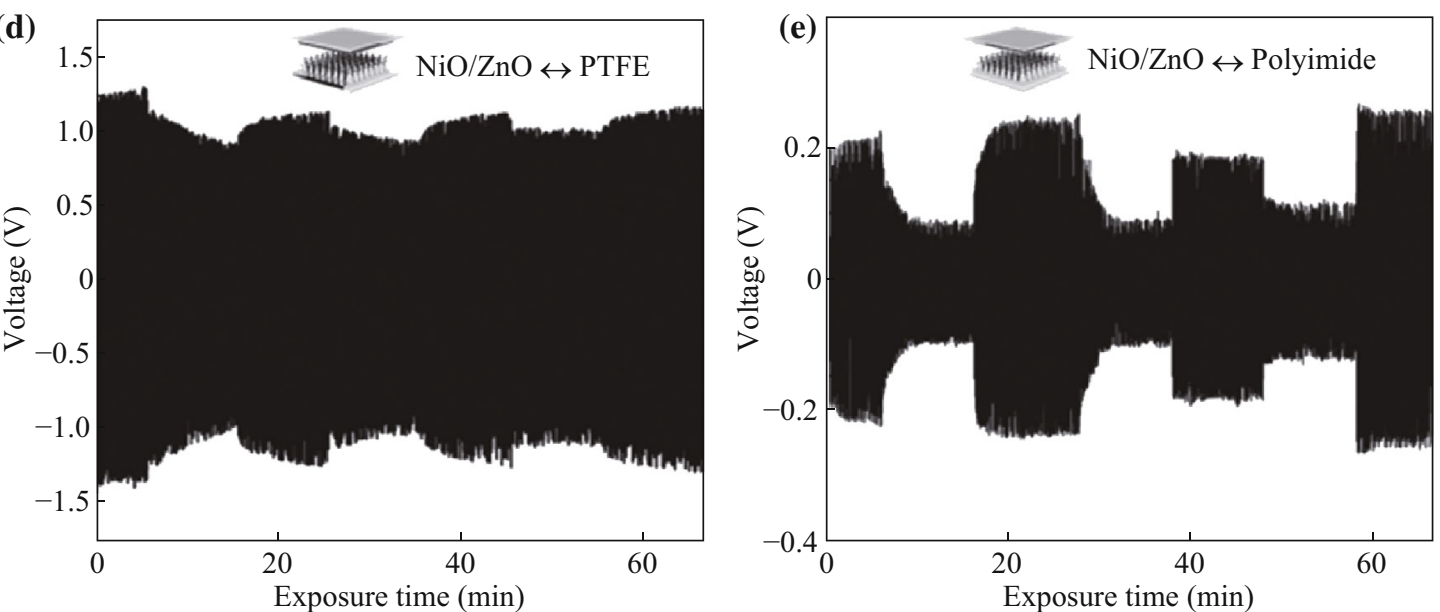

Fig. 8 A self-powered, room-temperature electronic nose based on triboelectrification and heterogeneous catalytic reaction. a Schematic illustration of the fabrication process of a self-powered electronic nose and an optical image of the as-fabricated device. SEM images of $\mathbf{b}$ the $\mathrm{ZnO} \mathrm{NWs}$ and $\mathbf{c}$ the $\mathrm{NiO}$ nanoparticle-decorated $\mathrm{ZnO} \mathrm{NWs}$ grown on the $\mathrm{SiO}_{2} / \mathrm{Si}$ (Scale bar, $500 \mathrm{~nm}$ ). The output performance of the sensor elements comprising $\mathbf{d} \mathrm{NiO}$ nanoparticle-decorated $\mathrm{ZnO}$-PFTE and e $\mathrm{NiO}$ nanoparticle-decorated $\mathrm{ZnO}$-PI under cycled compressive force of $50 \mathrm{~N}$ at an applied frequency of $4 \mathrm{~Hz}$. Figures adapted from [96]

consists of a gas flow processor, a smelling e-skin and a visualization panel. The visualization panel can directly display breath-alcohol concentration by counting the alight LED (Fig. 10h). The gas flow-induced output current/voltage is significantly dependent on the environmental atmosphere, which can act as olfactory bionic electric impulse. Against ethanol, the detection limit of the e-skin is $30 \mathrm{ppm}$, and the response is up to 66.8 against $210 \mathrm{ppm}$ ethanol gas flow. Interestingly, the response of the e-skin keeps stable under different gas flow rates or with different device sizes/bending status. Also, the e-skin has relatively short response/recovery time $(<25 \mathrm{~s})$ and can detect various volatile organic compounds (Fig. 10i). When an adult without drinking alcohol blows the system, all the 8 LEDs can be lighted. When a drunken adult blows the system, the ethanol in the breath can dramatically decrease the output of the e-skin, and the alight LEDs are dependent on the ethanol concentration (Fig. 10j). Finally, an application of the flexible smelling e-skin for visually identifying drunken driver without any external electricity power has been demonstrated [98].

The present results shed light on designing new specialized-function e-skin and novel self-powered nanosystem. Some other non-ZnO materials have also been studied for TENG-based self-powered gas sensing system, such as Pd-functionalized ITO surface and PET film for selfpowered $\mathrm{H}_{2}$ sensor [93], PDMS and PEDOT:PSS film with nylon fiber film for self-powered active acetylene gas sensing [99].

\subsection{Novel Mutual Independent System}

The approach of realization of self-powered sensors normally is to actively generate electrical signal by itself as a response to the stimulation or triggering from the ambient environment, as described in above-mentioned works. 

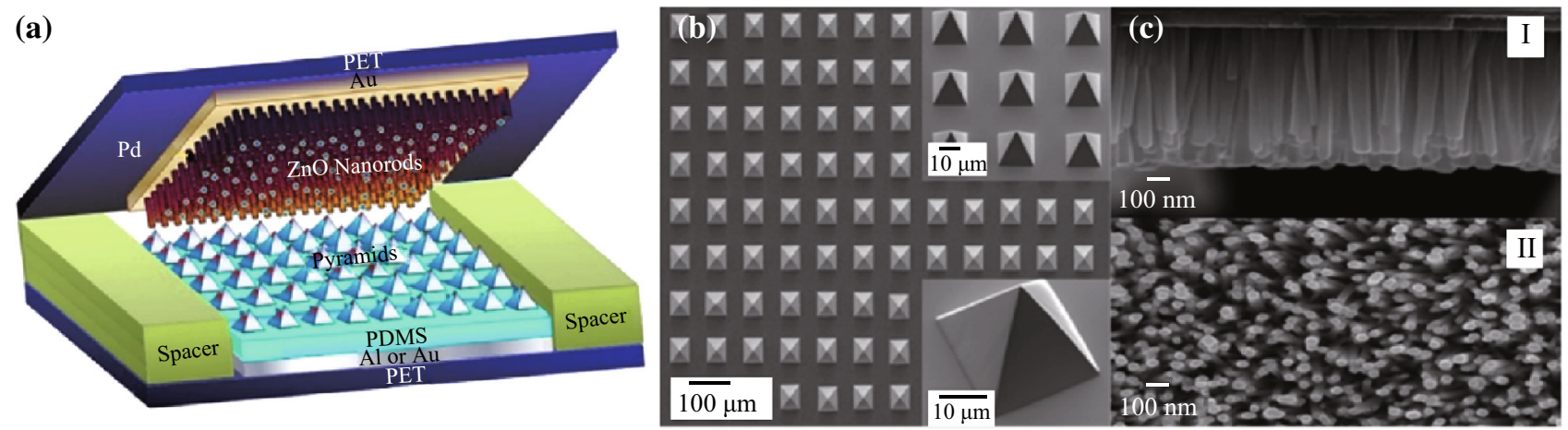

(d)

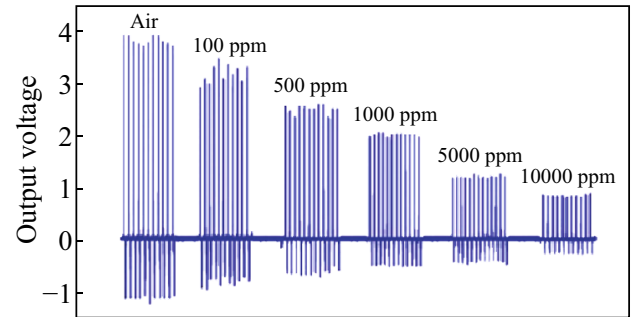

Time $(\mathrm{sec})$ (e)

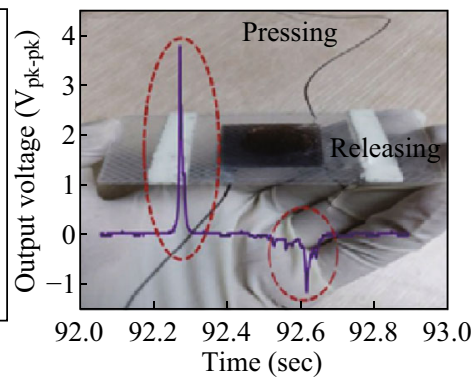

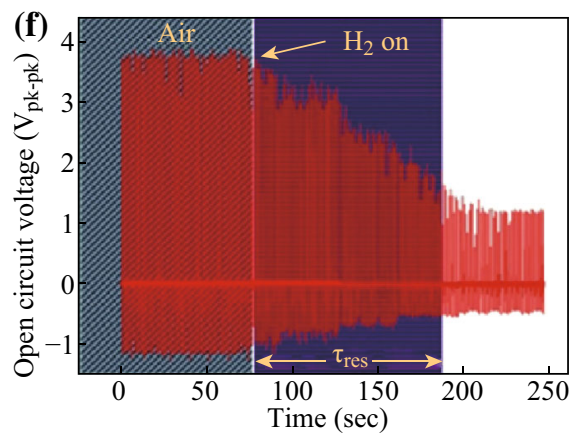

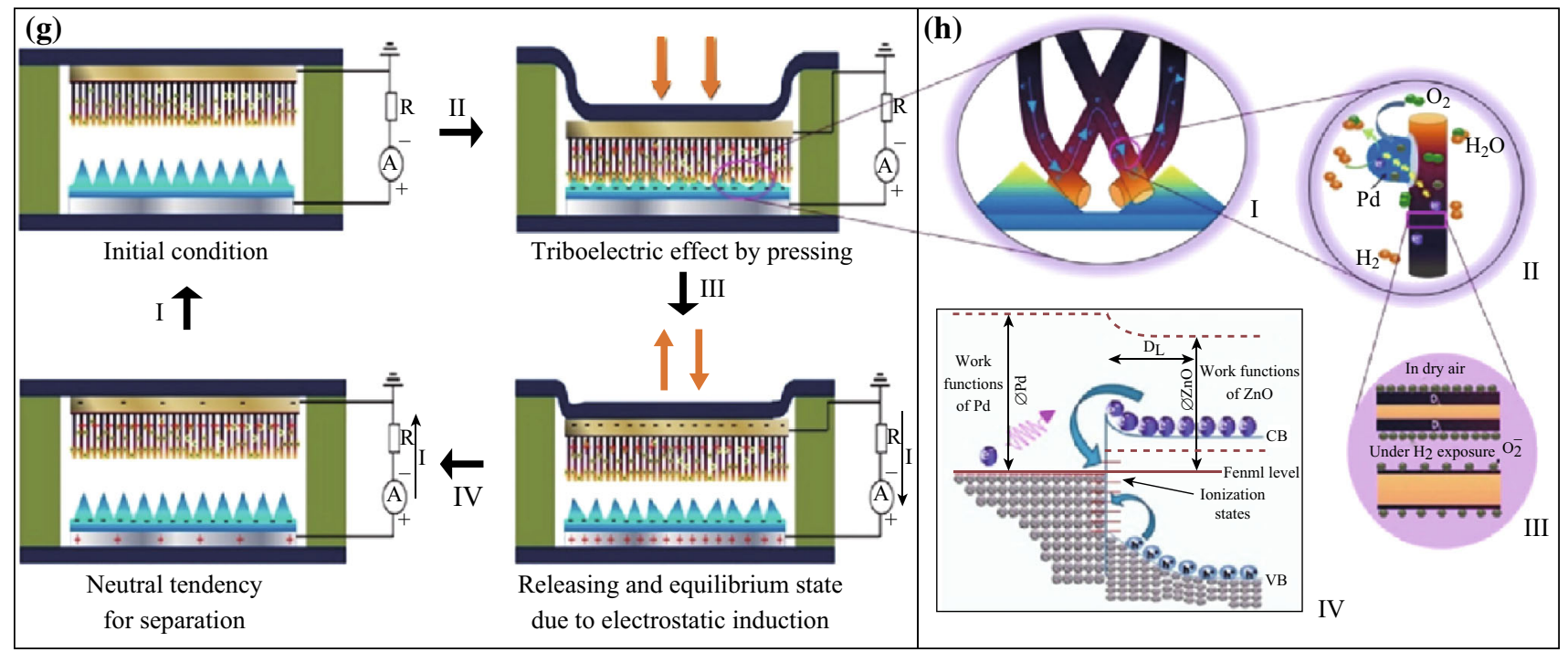

Fig. 9 A self-powered active hydrogen gas sensor with fast response at room temperature based on triboelectric effect. a Schematic of the asfabricated triboelectric-based $\mathrm{H}_{2}$ sensor (TEHS). b FESEM images of the micropatterned PDMS surface (the insets show the enlarged view of the micropyramids PDMS). $\mathbf{c}$ Cross-section (I) and in-plane (II) micrographs of $\mathrm{ZnO}$ nanoparticles. d Variation of open-circuit voltage with and without $\mathrm{H}_{2}$ exposure. e The pressing and releasing signal peaks of the TEHS. $\mathbf{f}$ Response time characteristics of the TEHS. Schematic of the TEHS's $\mathbf{g}$ triboelectric and $\mathbf{h}$ sensing mechanisms. Figures adapted from [97]

However, it still requires external monitoring circuits to collect the signal generated by NGs, which means these sensing systems are not genuinely and authentically "selfpowered" [19, 100, 101]. Obviously, developing appropriate NGs to directly charge or monitor sensors is another approach to achieve real "self-powered" system.

Facing this problem, Zhong Lin Wang's group, for the first time, introduced a completely new working principle of self-powered gas sensing system by fabricating a blow- driven TENG to supply power for active alcohol detection, as shown in Fig. 11. The basic structure of the self-powered gas sensing system mainly consists of three functional parts, a traditional gas sensor, an alarm as well as a blowdriven TENG as power source, which can be driven by mouth blowing (Fig. 11a, b). The vertically aligned fluorinated ethylene propylene (FEP) NWs with the average clustering diameter of $\sim 100 \mathrm{~nm}$ and length of $\sim 1 \mu \mathrm{m}$ have been prepared for enhancing triboelectric output 


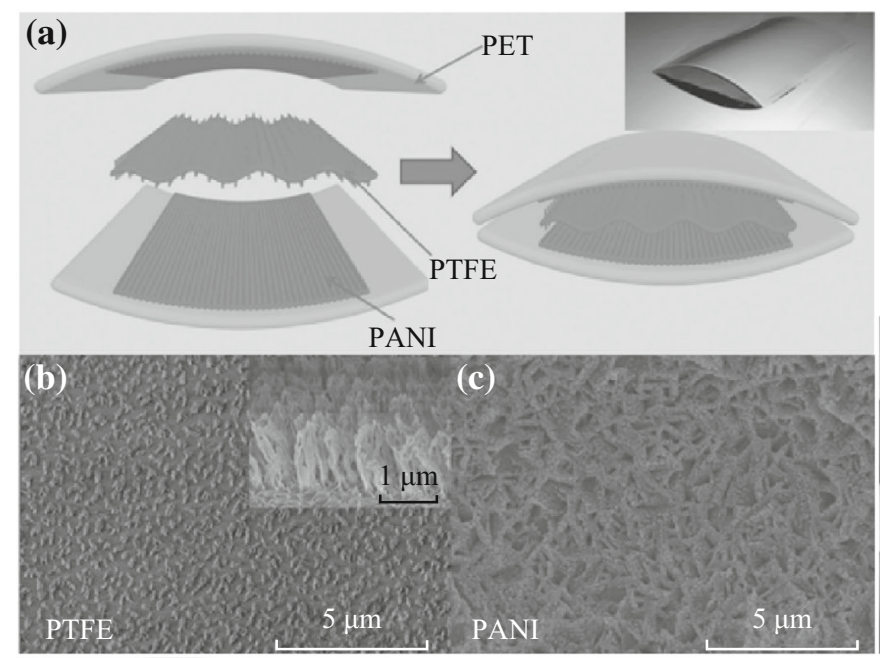

(h)
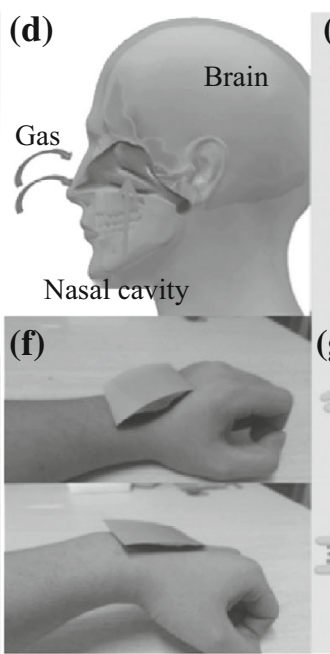

(e)
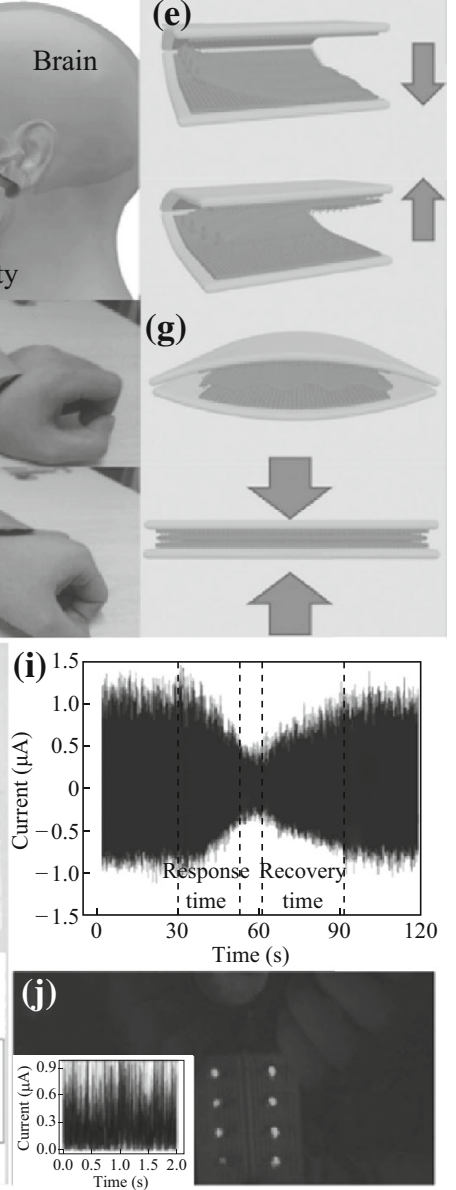

Fig. 10 Outputting olfactory bionic electric impulse by PANI/PTFE/PANI sandwich nanostructures and their application as flexible, smelling electronic skin. a Structure and fabrication process of the flexible smelling e-skin (the inset shows the photograph of the e-skin). b SEM image of PTFE nanostructures on the top view (the inset shows cross-sectional SEM image of PTFE NWs). c SEM image of PANI nanostructures on the top view. $\mathbf{d}$ and $\mathbf{e}$ The e-skin can be easily driven by gas flow (human heavy breath or blowing). $\mathbf{f}$, $\mathbf{g}$ The e-skin can be easily driven by body motion (pressure). h Photograph of the self-powered breath-alcohol visual detecting system. i Real-time continuous responding/recovering process of the e-skin against 210 ppm ethanol gas flow. j An adult without drinking alcohol can light 8 LEDs. Figures adapted from [98]

(Fig. 11c). The stator, as one triboelectric material, is composed of two copper electrodes with complementary patterns, which have been separated by fine trenches in between (Fig. 11d). The alcohol vapor would dramatically increase the resistance of the sensor that led to an increased voltage drop across the sensor, when the blow-driven TENG was blown by a tester after alcohol drinking (Fig. 11e). The as-developed active alcohol breath analyzer based on the BD-TENG is featured as high detection response of about 34-100 ppm alcohol gas under an optimized sensor working temperature, fast response time of $11 \mathrm{~s}$ as well as a fast recovery of $20 \mathrm{~s}$ (Fig. 11f). A signal processing circuit diagram of a complete self-securing warning system which can be triggered by an increased voltage signal is shown in Fig. 11g.

The induced voltage across the sensor holds a proportional relationship with the breathed-out alcohol concentration regardless of the blow speed and quality airflow so that this blow-driven TENG-based self-powered gas sensor makes a significant progress toward the practical application. All the results indicate that the novel self-powered sensing system enables to work stably and reliably. Besides, given other competitive features, including being light weight, easy fabrication, cost-effectiveness, the justified concept in this work not only launches a new approach with extensive potential in the field of gas sensing, but also make a significant progress toward the practical application of a novel mutual independent TENGbased self-powered gas sensing system [102].

\section{Conclusions and Outlook}

In this review, the establishment and latest progress in NGbased self-powered gas sensors are systematically summarized. We have also summarized recent researches about 
(a)

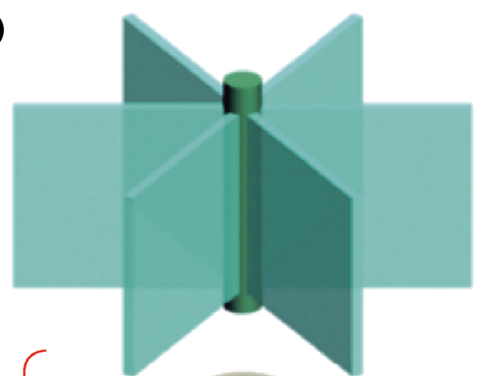

Acrylic

Bearing

TFEP

Elastic

Cu electrode
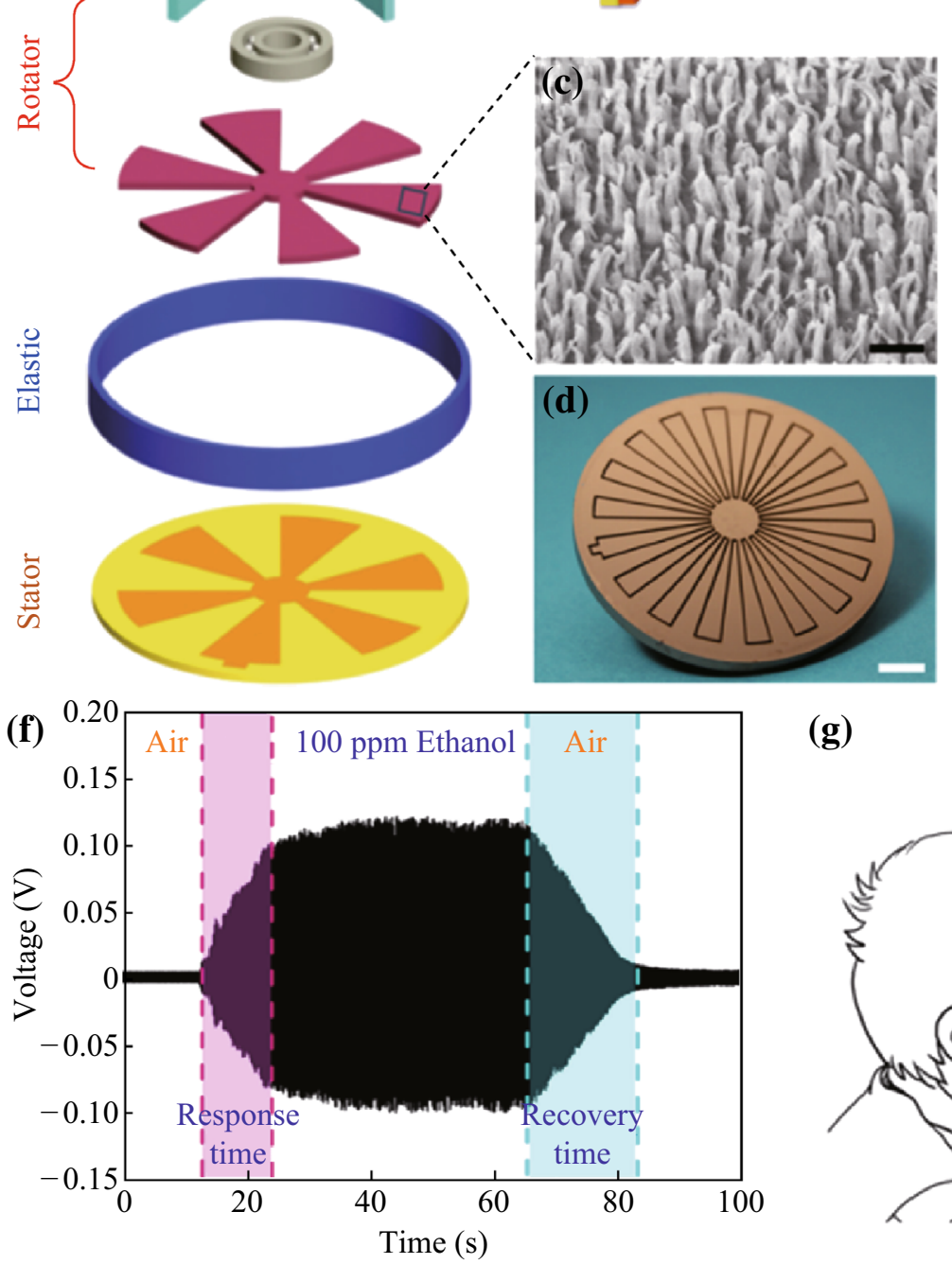

(g) (b)
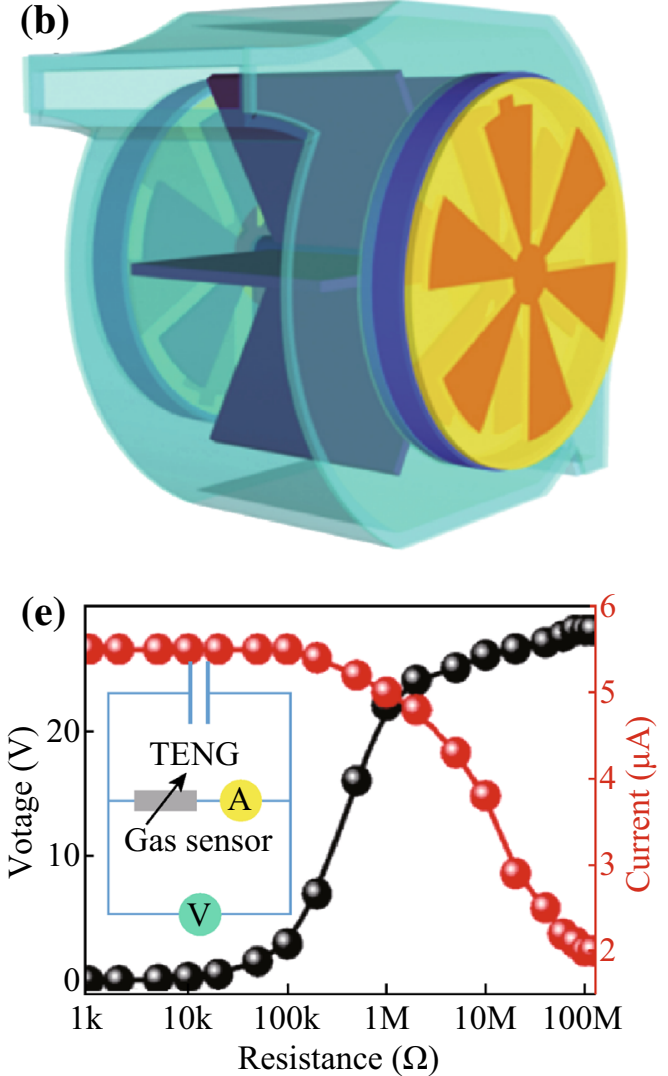
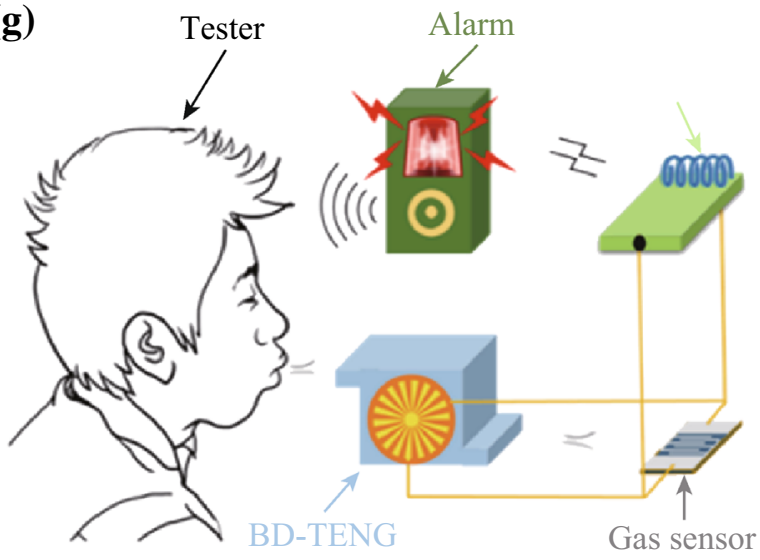

Fig. 11 Blow-driven TENG as an active alcohol breathalyzer. a Schematic illustrations of the functional components of the blow-driven triboelectric nanogenerator, which mainly consists of three parts, a rotator, a stator and a soft elastic. b A SEM image of the FEP polymer NWs (scale bar, $500 \mathrm{~nm}$ ). c A photograph of the as-fabricated stator (scale bar, $1 \mathrm{~cm}$ ). d A schematic illustration of an as-fabricated device (scale bar, $2 \mathrm{~cm}$ ). e The variation of the output voltage and current with external load resistances under a fixed blowing speed of $6 \mathrm{~m} / \mathrm{s}$ (the inset shows the measurement circuit diagram). f A real-time continuously measured voltage profile to show the dynamic response to ambient alcohol concentrations. g A schematic illustration showing the self-power breathalyzer. Figures adapted from [102]

various NG-based self-powered gas sensors in Table 1 . As an important functional application, self-powered gas sensors can operate independently based on two main technologies including PENG and TENG, without the use of external electricity storage/supply systems.
For PENG, it has been achieved in a series of works through the coupling of piezoelectric effect and gas sensing characteristics, in which the piezoelectric output of PENG acts as both the power source and sensing signal. The gas adsorption on the surface of piezoelectric-semiconductor 
Table 1 A summary of various NG-based self-powered gas sensors

\begin{tabular}{|c|c|c|c|c|c|c|}
\hline Type & Materials & Morphology & Gas & Concentration & Sensitivity & References \\
\hline Piezo & $\mathrm{ZnO}$ & NW & $\mathrm{H}_{2} \mathrm{~S}$ & $100 \mathrm{ppm}$ & $13.1^{\mathrm{A}}$ & [59] \\
\hline Piezo & $\mathrm{ZnO}$ & NS & Humidity & $10 \mathrm{ppm}$ & $2.96^{\mathrm{A}}$ & {$[60]$} \\
\hline Piezo & $\mathrm{ZnO}+\mathrm{UV}$ & NW & Ethanol & $700 \mathrm{ppm}$ & $85^{\mathrm{A}}$ & [103] \\
\hline Piezo & $\mathrm{Cu} / \mathrm{ZnO}$ & NW & $\mathrm{H}_{2} \mathrm{~S}$ & $1000 \mathrm{ppm}$ & $1045.76^{\mathrm{B}}$ & [66] \\
\hline Piezo & $\mathrm{Pd} / \mathrm{ZnO}$ & Nanoarray & Ethanol & 800 ppm & $108^{\mathrm{B}}$ & {$[67]$} \\
\hline Piezo & $\mathrm{Au} / \mathrm{ZnO}$ & NW Array & Ethanol & $1200 \mathrm{ppm}$ & $72.1^{\mathrm{A}}$ & {$[68]$} \\
\hline Piezo & $\mathrm{Cd} / \mathrm{ZnO}$ & NW & Humidity & $70 \% \mathrm{RH}$ & $85.7^{\mathrm{A}}$ & {$[70]$} \\
\hline Piezo & $\mathrm{Pt} / \mathrm{ZnO}$ & Nanoarray & Ethanol & $1000 \mathrm{ppm}$ & $37.14^{\mathrm{B}}$ & [69] \\
\hline Piezo & $\mathrm{SnO}_{2} / \mathrm{ZnO}$ & Nanoarray & $\mathrm{H}_{2}$ & 800 ppm & $471.4^{\mathrm{B}}$ & [104] \\
\hline Piezo & $\mathrm{ZnSnO}_{3} / \mathrm{ZnO}$ & NW & $\begin{array}{l}\text { Liquefied } \\
\text { petroleum }\end{array}$ & 8000 ppm & $83.23^{\mathrm{A}}$ & [105] \\
\hline Piezo & $\alpha-\mathrm{Fe}_{2} \mathrm{O}_{3} / \mathrm{ZnO}$ & NW Array & Ethanol & $700 \mathrm{ppm}$ & $706.8^{\mathrm{B}}$ & [106] \\
\hline Piezo & $\mathrm{NiO} / \mathrm{ZnO}$ & NW & $\mathrm{H}_{2} \mathrm{~S}$ & $1000 \mathrm{ppm}$ & $84.3^{\mathrm{B}}$ & [107] \\
\hline Piezo & $\mathrm{In}_{2} \mathrm{O}_{3} / \mathrm{ZnO}$ & Nanoarray & $\mathrm{H}_{2} \mathrm{~S}$ & 700 ppm & $925^{\mathrm{B}}$ & [108] \\
\hline Piezo & $\mathrm{CuO} / \mathrm{ZnO}$ & Nanoarray & $\mathrm{H}_{2} \mathrm{~S}$ & 800 ppm & $629.8^{\mathrm{B}}$ & [71] \\
\hline Piezo & $\mathrm{CeO}_{2} / \mathrm{ZnO}$ & Nanoarray & Humidity & $95 \% \mathrm{RH}$ & $82.1^{\mathrm{A}}$ & [109] \\
\hline Piezo & $p-\mathrm{Si} / n-\mathrm{ZnO}$ & NR & $\mathrm{NO}_{2}$ & $\begin{array}{l}750 \mathrm{ppb} \text { (amine) } \\
750 \mathrm{ppb} \text { (thiol) }\end{array}$ & $\begin{array}{l}23.5(\text { amine })^{\mathrm{A}} \\
12.8(\text { thiol })^{\mathrm{A}}\end{array}$ & [72] \\
\hline Piezo & $\mathrm{CdS}$ & NR & $\mathrm{H}_{2} \mathrm{~S}$ & $600 \mathrm{ppm}$ & $62.5^{\mathrm{A}}$ & {$[73]$} \\
\hline Tribo & $\begin{array}{l}\mathrm{NiO}-\mathrm{ZnO} \\
\text { PDMS }\end{array}$ & $\begin{array}{l}\text { NW } \\
\text { Film }\end{array}$ & Ethanol & $0.1 \%$ & $37.5^{\mathrm{A}}$ & [96] \\
\hline Tribo & $\begin{array}{l}\mathrm{Pd} / \mathrm{ZnO} \\
\mathrm{PDMS}\end{array}$ & $\begin{array}{l}\text { NW } \\
\text { Film }\end{array}$ & $\mathrm{H}_{2}$ & $10,000 \mathrm{ppm}$ & $373^{\mathrm{B}}$ & [97] \\
\hline Tribo & $\begin{array}{l}\text { PANI } \\
\text { PTFE }\end{array}$ & $\begin{array}{l}\text { Film } \\
\text { Film }\end{array}$ & Ethanol & $210 \mathrm{ppm}$ & $66.8^{\mathrm{C}}$ & [98] \\
\hline Tribo & $\begin{array}{l}\text { PEDOT:PSS } \\
\text { Ag-ZnO/nylon }\end{array}$ & $\begin{array}{l}\text { Film } \\
\text { Fiber film }\end{array}$ & $\mathrm{C}_{2} \mathrm{H}_{2}$ & $1000 \mathrm{ppm}$ & $\begin{array}{l}70.9 \text { (indoor) }^{\mathrm{A}} \\
89 \text { (outdoor) }^{\mathrm{A}}\end{array}$ & [99] \\
\hline Tribo & $\begin{array}{l}\text { Pd-ITO } \\
\text { PET }\end{array}$ & $\begin{array}{l}\text { Film } \\
\text { film }\end{array}$ & $\mathrm{H}_{2}$ & $1 \%$ & $75^{\mathrm{A}}$ & {$[93]$} \\
\hline Tribo & $\begin{array}{l}\mathrm{Pd} / \mathrm{ZnO} \\
\mathrm{PDMS}\end{array}$ & $\begin{array}{l}\text { NW } \\
\text { Film }\end{array}$ & $\mathrm{H}_{2}$ & 3 vol\% & $1457.69^{\mathrm{B}}$ & [94] \\
\hline Tribo & $\begin{array}{l}\mathrm{Co}_{3} \mathrm{O}_{4} \\
\mathrm{Cu} \text { and PTFE }\end{array}$ & $\begin{array}{l}\text { NR } \\
\text { Film }\end{array}$ & Ethanol & $100 \mathrm{ppm}$ & $34^{\mathrm{D}}$ & {$[102]$} \\
\hline
\end{tabular}

A: $S \%=\left(V_{\mathrm{a}}-V_{\mathrm{g}}\right) / V_{\mathrm{a}} \times 100 \% ; \mathrm{B}: S \%=\left(V_{\mathrm{a}}-V_{\mathrm{g}}\right) / V_{\mathrm{g}} \times 100 \% ; \mathrm{C}: S \%=\left(I_{\mathrm{a}}-I_{\mathrm{g}}\right) / I_{\mathrm{a}} \times 100 \% ; \mathrm{D}:$ $S=V_{\mathrm{a}} / V_{\mathrm{g}}$, where $V_{\mathrm{a}}$ and $V_{\mathrm{g}}$ are the output voltages of the device under the same conditions in dry air and the test gas, respectively; $I_{\mathrm{a}}$ and $I_{\mathrm{g}}$ represent the output current in air and test gas, respectively materials can change the free-carrier density, which can vary the piezoelectric output upon applied deformation through the piezoelectric screening effect. However, compared with the traditional gas sensors, the conventional PENG-based self-powered gas sensors have some limitations. Firstly, the requirements of materials both having piezoelectric and semiconducting gas sensing properties, mainly referring to $\mathrm{ZnO}$ as building block, seriously restrict the most use of other non-piezoelectric but owning excellent gas sensing materials. Introducing noble metal decorated material, heterostructure and organic material-based gas sensors will further expand the scope for self-powered gas sensing systems. Secondly, due to their compact and completely sealed device structures, the response time of these gas sensors is in the range of tens of seconds, which is not fully well-suited for gas exposure on the sensing materials. Therefore, the complexities of the requirements demand to search for some simple sensor packaging system for better sensor performances. Thirdly, in most cases, the adsorption of gas molecules will happen for more than one kind, a better method of ascribing the variation of the piezoelectric output to a specific gas or optimizing its selectivity should be considered. Fourthly, theoretically, high piezoelectric potential would be generated when a large external force is applied onto a nanowire with a small thickness, because large deflection can be produced. 
However, in real cases, the deflection of nanodevice is restricted by their mechanical strength and flexibility.

While TENG-based self-powered gas sensors work simply by the formation of a dipole layer after triboelectric contact and static separation between two materials of the triboelectric series and the triboelectric charge density is greatly influenced by the surface alteration of certain chemisorptions of the molecular oxygen species. In comparison with PENG, even though most gas sensing materials are not in the tribo-series list, $\mathrm{ZnO}, \mathrm{SnO}_{2}$, etc. exhibit triboelectric properties due to their finite conductivity characteristics, which are widening the range of choosing tribo-gas sensing materials. Meanwhile, a variety of structural designs also solve the problem of gas exposure on the sensing materials. However, it also exists some limitations, such as firstly, the introduction of motion will cause the mechanical disturbance of measuring equipment; Secondly, the output of TENG is unstable, and it may change with respect to the environment when used for realtime self-powered systems; thirdly, it still requires external monitoring circuits to collect the signal generated by NGs, which means these sensing systems are not genuinely and authentically "self-powered." Developing appropriate TENGs to directly charge or monitor sensors is the trend of self-powered gas sensors. The whole system does not require external monitoring circuits to collect the signal, which have achieved genuinely and authentically "selfpowered." Meanwhile, the three main parts of this system work independently and do not interfere with each other. Moreover, the voltage output of TENG remains constant under various working frequencies. All the results indicate that the novel system enables to work stably and reliably, which provides a strong theoretical basis and technical support for the next generation of self-powered gas sensing system.

As a new field by coupling piezoelectric or triboelectric with semiconducting gas sensing characteristics, the NGbased self-powered gas sensing system has been demonstrated with sustainable, flexible, light weight, high efficient, cost-effective and environmental friendly designs. Continued progress in this field will lead to a class of selfpowered gas sensors with superior sensitivity, excellent selectivity, high reliability and extended lifetimes for a wide range of environments and applications. It will be a collaborative developing field with various disciplines such as materials, energy, chemistry, automation, mechatronics and information.

Acknowledgements The work was supported by Natural Science Foundation of China (NSFC) (Grant No. U1432249) and the Priority Academic Program Development of Jiangsu Higher Education Institutions (PAPD). This is also a project supported by Collaborative Innovation Center of Suzhou Nano Science \& Technology and sponsored by Qing Lan Project.
Open Access This article is distributed under the terms of the Creative Commons Attribution 4.0 International License (http://creative commons.org/licenses/by/4.0/), which permits unrestricted use, distribution, and reproduction in any medium, provided you give appropriate credit to the original author(s) and the source, provide a link to the Creative Commons license, and indicate if changes were made.

\section{References}

1. L. Atzori, A. Iera, G. Morabito, The internet of things: a survey. Comput. Netw. 54(15), 2787-2805 (2010). doi:10.1016/j.com net.2010.05.010

2. D. Bandyopadhyay, J. Sen, Internet of things: applications and challenges in technology and standardization. Wireless Pers. Commun. 58(1), 49-69 (2011). doi:10.1007/s11277-011-0288-5

3. Z.M. Fadlullah, M.M. Fouda, N. Kato, A. Takeuchi, N. Iwasaki, Y. Nozaki, Toward intelligent machine-to-machine communications in smart grid. IEEE Commun. Mag. 49(4), 60-65 (2011). doi:10.1109/MCOM.2011.5741147

4. D. Niyato, L. Xiao, P. Wang, Machine-to-machine communications for home energy management system in smart grid. IEEE Commun. Mag. 49(4), 53-59 (2011). doi:10.1109/ MCOM.2011.5741146

5. Z.L. Wang, Self-powered nanosensors and nanosystems. Adv. Mater. 24(2), 280-285 (2012). doi:10.1002/adma.201102958

6. Z.L. Wang, G. Zhu, Y. Yang, S. Wang, C. Pan, Progress in nanogenerators for portable electronics. Mater. Today 15(12), 532-543 (2012). doi:10.1016/S1369-7021(13)70011-7

7. P. Glynne-Jones, N.M. White, Self-powered systems: a review of energy sources. Sens. Rev. 21(2), 91-98 (2001). doi:10.1108/ 02602280110388252

8. J. Jun, B. Chou, J. Lin, A. Phipps, X. Shengwen et al., A hydrogen leakage detection system using self-powered wireless hydrogen sensor nodes. Solid State Electron. 51(7), 1018-1022 (2007). doi:10.1016/j.sse.2007.05.019

9. R. Torah, P. Glynne-Jones, M. Tudor, T. O’Donnell, S. Roy, S. Beeby, Self-powered autonomous wireless sensor node using vibration energy harvesting. Meas. Sci. Technol. 19(12), 125202 (2008). doi:10.1088/0957-0233/19/12/125202

10. Z.L. Wang, J. Song, Piezoelectric nanogenerators based on zinc oxide nanowire arrays. Science 312(5771), 242-246 (2006). doi:10.1126/science.1124005

11. F.R. Fan, Z.Q. Tian, Z.L. Wang, Flexible triboelectric generator. Nano Energy 1(2), 328-334 (2012). doi:10.1016/j.nanoen.2012. 01.004

12. X.S. Zhang, M.D. Han, B. Meng, H.X. Zhang, High performance triboelectric nanogenerators based on large-scale massfabrication technologies. Nano Energy 11, 304-322 (2015). doi:10.1016/j.nanoen.2014.11.012

13. Q. Liang, Q. Zhang, X. Yan, X. Liao, L. Han, F. Yi, M. Ma, Y. Zhang, Recyclable and green triboelectric nanogenerator. Adv. Mater. 29(5), 1604961 (2017). doi:10.1002/adma.201604961

14. Z.L. Wang, On Maxwell's displacement current for energy and sensors: the origin of nanogenerators. Mater. Today (article in press) (2017). doi:10.1016/j.mattod.2016.12.001

15. X. Cao, Y. Jie, N. Wang, Z.L. Wang, Triboelectric nanogenerators driven self-powered electrochemical processes for energy and environmental science. Adv. Energy Mater. 6(23), 1600665 (2016). doi:10.1002/aenm.201600665

16. J. Wang, Z. Wen, Y. Zi, L. Lin, C. Wu, H. Guo, Y. Xi, Y. Xu, Z.L. Wang, Self-powered electrochemical synthesis of polypyrrole from the pulsed output of a triboelectric nanogenerator as a sustainable energy system. Adv. Funct. Mater. 26(20), 3542-3548 (2016). doi:10.1002/adfm.201600021 
17. Z. Li, J. Chen, J. Zhou, L. Zheng, K.C. Pradel et al., Highefficiency ramie fiber degumming and self-powered degumming wastewater treatment using triboelectric nanogenerator. Nano Energy 22, 548-557 (2016). doi:10.1016/j.nanoen.2016.03.002

18. H. Guo, Z. Wen, Y. Zi, M.H. Yeh, J. Wang, L. Zhu, C. Hu, Z.L. Wang, A water-proof triboelectric-electromagnetic hybrid generator for energy harvesting in harsh environments. Adv. Energy Mater. 6(6), 1501593 (2016). doi:10.1002/aenm. 201501593

19. Z.L. Wang, Triboelectric nanogenerators as new energy technology for self-powered systems and as active mechanical and chemical sensors. ACS Nano 7(11), 9533-9557 (2013). doi:10. $1021 / \mathrm{nn} 404614 \mathrm{z}$

20. P.K. Yang, Z.H. Lin, K.C. Pradel, L. Lin, X. Li, X. Wen, J.H. He, Z.L. Wang, Paper-based origami triboelectric nanogenerators and self-powered pressure sensors. ACS Nano 9(1), 901-907 (2015). doi:10.1021/nn506631t

21. K.Y. Lee, H.J. Yoon, T. Jiang, X. Wen, W. Seung, S.W. Kim, Z.L. Wang, Fully packaged self-powered triboelectric pressure sensor using hemispheres-array. Adv. Energy Mater. 6(11), 1502566 (2016). doi:10.1002/aenm.201502566

22. Y.S. Zhou, G. Zhu, S. Niu, Y. Liu, P. Bai, Q. Jing, Z.L. Wang, Nanometer resolution self-powered static and dynamic motion sensor based on micro-grated triboelectrification. Adv. Mater. 26(11), 1719-1724 (2014). doi:10.1002/adma.201304619

23. Q. Jing, Y. Xie, G. Zhu, R.P. Han, Z.L. Wang, Self-powered thin-film motion vector sensor. Nat. Commun. 6, 8031 (2015). doi:10.1038/ncomms9031

24. J. Yang, J. Chen, Y. Liu, W. Yang, Y. Su, Z.L. Wang, Triboelectrification-based organic film nanogenerator for acoustic energy harvesting and self-powered active acoustic sensing. ACS Nano 8(3), 2649-2657 (2014). doi:10.1021/nn4063616

25. X. Fan, J. Chen, J. Yang, P. Bai, Z. Li, Z.L. Wang, Ultrathin, rollable, paper-based triboelectric nanogenerator for acoustic energy harvesting and self-powered sound recording. ACS Nano 9(4), 4236-4243 (2015). doi:10.1021/acsnano.5b00618

26. J. Chen, G. Zhu, J. Yang, Q. Jing, P. Bai, W. Yang, X. Qi, Y. Su, Z.L. Wang, Personalized keystroke dynamics for self-powered human-machine interfacing. ACS Nano 9(1), 105-116 (2015). doi: $10.1021 / \mathrm{nn} 506832 \mathrm{w}$

27. G. Zhu, W.Q. Yang, T. Zhang, Q. Jing, J. Chen, Y.S. Zhou, P. Bai, Z.L. Wang, Self-powered, ultrasensitive, flexible tactile sensors based on contact electrification. Nano Lett. 14(6), 3208-3213 (2014). doi:10.1021/n15005652

28. J. Yang, J. Chen, Y. Su, Q. Jing, Z. Li, F. Yi, X. Wen, Z. Wang, Z.L. Wang, Eardrum-inspired active sensors for self-powered cardiovascular system characterization and throat-attached antiinterference voice recognition. Adv. Mater. 27(8), 1316-1326 (2015). doi:10.1002/adma.201404794

29. Q. Zheng, Y. Zou, Y. Zhang, Z. Liu, B. Shi et al., Biodegradable triboelectric nanogenerator as a life-time designed implantable power source. Sci. Adv. 2(3), e1501478 (2016). doi:10.1126/sciadv. 1501478

30. Y. Ma, Q. Zheng, Y. Liu, B. Shi, X. Xue et al., Self-powered, one-stop, and multifunctional implantable triboelectric active sensor for real-time biomedical monitoring. Nano Lett. 16(10), 6042-6051 (2016). doi:10.1021/acs.nanolett.6b01968

31. Q. Zheng, H. Zhang, B. Shi, X. Xue, Z. Liu et al., In vivo selfpowered wireless cardiac monitoring via implantable triboelectric nanogenerator. ACS Nano 10(7), 6510-6518 (2016). doi:10. 1021/acsnano.6b02693

32. W. Tang, Y. Han, C.B. Han, C.Z. Gao, X. Cao, Z.L. Wang, Selfpowered water splitting using flowing kinetic energy. Adv. Mater. 27(2), 272-276 (2015). doi:10.1002/adma.201404071

33. Y. Yang, H. Zhang, Z.H. Lin, Y. Liu, J. Chen, Z. Lin, Y.S. Zhou, C.P. Wong, Z.L. Wang, A hybrid energy cell for self- powered water splitting. Energy Environ. Sci. 6(8), 2429-2434 (2013). doi:10.1039/c3ee41485j

34. S. Chen, C. Gao, W. Tang, H. Zhu, Y. Han, Q. Jiang, T. Li, X. Cao, Z. Wang, Self-powered cleaning of air pollution by wind driven triboelectric nanogenerator. Nano Energy 14, 217-225 (2015). doi:10.1016/j.nanoen.2014.12.013

35. Y. Yang, H. Zhang, S. Lee, D. Kim, W. Hwang, Z.L. Wang, Hybrid energy cell for degradation of methyl orange by selfpowered electrocatalytic oxidation. Nano Lett. 13(2), 803-808 (2013). doi:10.1021/n13046188

36. W. Guo, X. Li, M. Chen, L. Xu, L. Dong et al., Electrochemical cathodic protection powered by triboelectric nanogenerator. Adv. Funct. Mater. 24(42), 6691-6699 (2014). doi:10.1002/ adfm. 201401168

37. X. Li, J. Tao, W. Guo, X. Zhang, J. Luo, M. Chen, J. Zhu, C. Pan, A self-powered system based on triboelectric nanogenerators and supercapacitors for metal corrosion prevention. J. Mater. Chem. A 3(45), 22663-22668 (2015). doi:10.1039/ C5TA07053H

38. X. Yang, G. Zhu, S. Wang, R. Zhang, L. Lin, W. Wu, Z.L. Wang, A self-powered electrochromic device driven by a nanogenerator. Energy Environ. Sci. 5(11), 9462-9466 (2012). doi:10.1039/c2ee23194h

39. M.H. Yeh, L. Lin, P.K. Yang, Z.L. Wang, Motion-driven electrochromic reactions for self-powered smart window system. ACS Nano 9(5), 4757-4765 (2015). doi:10.1021/acsnano. 5 b00706

40. Z. Li, J. Chen, J. Yang, Y. Su, X. Fan, Y. Wu, C. Yu, Z.L. Wang, $\beta-$ cyclodextrin enhanced triboelectrification for self-powered phenol detection and electrochemical degradation. Energy Environ. Sci. 8(3), 887-896 (2015). doi:10.1039/C4EE03596H

41. M.H. Yeh, H. Guo, L. Lin, Z. Wen, Z. Li, C. Hu, Z.L. Wang, Rolling friction enhanced free-standing triboelectric nanogenerators and their applications in self-powered electrochemical recovery systems. Adv. Funct. Mater. 26(7), 1054-1062 (2016). doi:10.1002/adfm.201504396

42. T. Wagner, S. Haffer, C. Weinberger, D. Klaus, M. Tiemann, Mesoporous materials as gas sensors. Chem. Soc. Rev. 42, 4036-4053 (2013). doi:10.1039/C2CS35379B

43. Z. Wen, L. Zhu, W. Mei, Y. Li, L. Hu, L. Sun, W. Wan, Z. Ye, A facile fluorine-mediated hydrothermal route to controlled synthesis of rhombus-shaped $\mathrm{Co}_{3} \mathrm{O}_{4}$ nanorod arrays and their application in gas sensing. J. Mater. Chem. A 1(25), 7511-7518 (2013). doi:10.1039/c3ta11004d

44. Z. Wen, L. Zhu, W. Mei, L. Hu, Y. Li, L. Sun, H. Cai, Z. Ye, Rhombus-shaped $\mathrm{Co}_{3} \mathrm{O}_{4}$ nanorod arrays for high-performance gas sensor. Sens. Actuators B 186, 172-179 (2013). doi:10. 1016/j.snb.2013.05.093

45. Z. Wen, L. Zhu, Y. Li, Z. Zhang, Z. Ye, Mesoporous $\mathrm{Co}_{3} \mathrm{O}_{4}$ nanoneedle arrays for high-performance gas sensor. Sens. Actuators B 203, 873-879 (2014). doi:10.1016/j.snb.2014.06. 124

46. Y. Gao, Z.L. Wang, Electrostatic potential in a bent piezoelectric nanowire. The fundamental theory of nanogenerator and nanopiezotronics. Nano Lett. 7(8), 2499-2505 (2007). doi:10. 1021/n1071310j

47. Y. Zi, L. Lin, J. Wang, S. Wang, J. Chen, X. Fan, P.K. Yang, F. Yi, Z.L. Wang, Triboelectric-pyroelectric-piezoelectric hybrid cell for high-efficiency energy-harvesting and self-powered sensing. Adv. Mater. 27(14), 2340-2347 (2015). doi:10.1002/ adma.201500121

48. S. Xu, Y. Qin, C. Xu, Y. Wei, R. Yang, Z.L. Wang, Selfpowered nanowire devices. Nat. Nano 5(5), 366-373 (2010). doi:10.1038/nnano.2010.46

49. S.M. Kim, H. Kim, Y. Nam, S. Kim, Effects of external surface charges on the enhanced piezoelectric potential of $\mathrm{ZnO}$ and $\mathrm{AlN}$ 
nanowires and nanotubes. AIP Adv. 2(4), 042174 (2012). doi:10.1063/1.4770314

50. T.Y. Wei, P.H. Yeh, S.Y. Lu, Z.L. Wang, Gigantic enhancement in sensitivity using Schottky contacted nanowire nanosensor. J. Am. Chem. Soc. 131(48), 17690-17695 (2009). doi:10.1021/ ja907585c

51. C. Zhang, Z.L. Wang, Tribotronics-A new field by coupling triboelectricity and semiconductor. Nano Today 11(4), 521-536 (2016). doi:10.1016/j.nantod.2016.07.004

52. M. Peng, Y. Liu, A. Yu, Y. Zhang, C. Liu et al., Flexible selfpowered $\mathrm{GaN}$ ultraviolet photoswitch with piezo-phototronic effect enhanced on/off ratio. ACS Nano 10(1), 1572-1579 (2016). doi:10.1021/acsnano.5b07217

53. N.R. Alluri, S. Selvarajan, A. Chandrasekhar, S. Balasubramaniam, J.H. Jeong, S.J. Kim, Self powered $\mathrm{pH}$ sensor using piezoelectric composite worm structures derived by ionotropic gelation approach. Sens. Actuators B 237, 534-544 (2016). doi:10.1016/j.snb.2016.06.134

54. Z. Wen, L. Zhu, L. Li, L. Sun, H. Cai, Z. Ye, A fluorinemediated hydrothermal method to synthesize mesoporous rhombic $\mathrm{ZnO}$ nanorod arrays and their gas sensor application. Dalton Trans. 42(44), 15551-15554 (2013). doi:10.1039/ c3dt51994e

55. Z. Wen, L. Zhu, Z. Zhang, Z. Ye, Fabrication of gas sensor based on mesoporous rhombus-shaped $\mathrm{ZnO}$ rod arrays. Sens. Actuators B 208, 112-121 (2015). doi:10.1016/j.snb.2014.11. 024

56. Q.J. Jiang, J.G. Lu, Y.L. Yuan, L.W. Sun, X. Wang, Z. Wen, Z.Z. Ye, D. Xiao, H.Z. Ge, Y. Zhao, Tailoring the morphology, optical and electrical properties of DC-sputtered $\mathrm{ZnO}$ : Al films by post thermal and plasma treatments. Mater. Lett. 106, 125-128 (2013). doi:10.1016/j.matlet.2013. 05.002

57. W. Wan, J. Huang, L. Zhu, L. Hu, Z. Wen, L. Sun, Z. Ye, Defects induced ferromagnetism in $\mathrm{ZnO}$ nanowire arrays doped with copper. CrystEngComm 15(39), 7887-7894 (2013). doi:10. 1039/c3ce40819a

58. W. Dai, X. Pan, S. Chen, C. Chen, Z. Wen, H. Zhang, Z. Ye, Honeycomb-like $\mathrm{NiO} / \mathrm{ZnO}$ heterostructured nanorods: photochemical synthesis, characterization, and enhanced UV detection performance. J. Mater. Chem. C 2(23), 4606-4614 (2014). doi:10.1039/c4tc00157e

59. X. Xue, Y. Nie, B. He, L. Xing, Y. Zhang, Z.L. Wang, Surface free-carrier screening effect on the output of a $\mathrm{ZnO}$ nanowire nanogenerator and its potential as a self-powered active gas sensor. Nanotechnology 24(22), 225501 (2013). doi:10.1088/ 0957-4484/24/22/225501

60. E. Modaresinezhad, S. Darbari, Realization of a room-temperature/self-powered humidity sensor, based on $\mathrm{ZnO}$ nanosheets. Sens. Actuators B 237, 358-366 (2016). doi:10.1016/j.snb.2016. 06.097

61. L. Zhang, Z. Gao, C. Liu, Y. Zhang, Z. Tu et al., Synthesis of $\mathrm{TiO}_{2}$ decorated $\mathrm{Co}_{3} \mathrm{O}_{4}$ acicular nanowire arrays and their application as an ethanol sensor. J. Mater. Chem. A 3(6), 2794-2801 (2015). doi:10.1039/C4TA06440B

62. Z. Zhang, Z. Wen, Z. Ye, L. Zhu, Gas sensors based on ultrathin porous $\mathrm{Co}_{3} \mathrm{O}_{4}$ nanosheets to detect acetone at low temperature. RSC Adv. 5(74), 59976-59982 (2015). doi:10.1039/ C5RA08536E

63. Z. Zhang, L. Zhu, Z. Wen, Z. Ye, Controllable synthesis of $\mathrm{Co}_{3} \mathrm{O}_{4}$ crossed nanosheet arrays toward an acetone gas sensor. Sens. Actuators B 238, 1052-1059 (2017). doi:10.1016/j.snb. 2016.07.154

64. G. Korotcenkov, Metal oxides for solid-state gas sensors: What determines our choice? Mater. Sci. Eng. B 139(1), 1-23 (2007). doi:10.1016/j.mseb.2007.01.044
65. C.X. Wang, L.W. Yin, L.Y. Zhang, D. Xiang, R. Gao, Metal oxide gas sensors: sensitivity and influencing factors. Sensors 10(3), 2088-2106 (2010). doi:10.3390/s100302088

66. Y. Fu, Y. Zhao, P. Wang, L. Xing, X. Xue, High response and selectivity of a $\mathrm{Cu}-\mathrm{ZnO}$ nanowire nanogenerator as a selfpowered/active $\mathrm{H}_{2} \mathrm{~S}$ sensor. Phys. Chem. Chem. Phys. 17(3), 2121-2126 (2015). doi:10.1039/C4CP04983G

67. Y. Lin, P. Deng, Y. Nie, Y. Hu, L. Xing, Y. Zhang, X. Xue, Room-temperature self-powered ethanol sensing of a $\mathrm{Pd} / \mathrm{ZnO}$ nanoarray nanogenerator driven by human finger movement. Nanoscale 6(9), 4604-4610 (2014). doi:10.1039/c3nr06809a

68. L. Xing, Y. Hu, P. Wang, Y. Zhao, Y. Nie, P. Deng, X. Xue, Realizing room-temperature self-powered ethanol sensing of $\mathrm{Au} / \mathrm{ZnO}$ nanowire arrays by coupling the piezotronics effect of $\mathrm{ZnO}$ and the catalysis of noble metal. Appl. Phys. Lett. 104(1), 013109 (2014). doi:10.1063/1.4861169

69. Y.Y. Zhao, X. Lai, P. Deng, Y.X. Nie, Y. Zhang, L.L. Xing, $\mathrm{X} . Y$. Xue, $\mathrm{Pt} / \mathrm{ZnO}$ nanoarray nanogenerator as self-powered active gas sensor with linear ethanol sensing at room temperature. Nanotechnology 25(11), 115502 (2014). doi:10.1088/09574484/25/11/115502

70. B. Yu, Y. Fu, P. Wang, Y. Zhao, L. Xing, X. Xue, Enhanced piezo-humidity sensing of a $\mathrm{Cd}-\mathrm{ZnO}$ nanowire nanogenerator as a self-powered/active gas sensor by coupling the piezoelectric screening effect and dopant displacement mechanism. Phys. Chem. Chem. Phys. 17(16), 10856-10860 (2015). doi:10.1039/ C5CP00893J

71. N. Yuxin, D. Ping, Z. Yayu, W. Penglei, X. Lili, Z. Yan, X. Xinyu, The conversion of $\mathrm{PN}$-junction influencing the piezoelectric output of a $\mathrm{CuO} / \mathrm{ZnO}$ nanoarray nanogenerator and its application as a room-temperature self-powered active $\mathrm{H}_{2} \mathrm{~S}$ sensor. Nanotechnology 25(26), 265501 (2014). doi:10.1088/ 0957-4484/25/26/265501

72. M.W.G. Hoffmann, L. Mayrhofer, O. Casals, L. Caccamo, F. Hernandez-Ramirez et al., A highly selective and self-powered gas sensor via organic surface functionalization of $\mathrm{p}-\mathrm{Si} / \mathrm{n}-\mathrm{Zno}$ diodes. Adv. Mater. 26(47), 8017-8022 (2014). doi:10.1002/ adma.201403073

73. W. Penglei, D. Ping, N. Yuxin, Z. Yayu, Z. Yan, X. Lili, X. Xinyu, Synthesis of CdS nanorod arrays and their applications in flexible piezo-driven active $\mathrm{H}_{2} \mathrm{~S}$ sensors. Nanotechnology 25(7), 075501 (2014). doi:10.1088/0957-4484/25/7/075501

74. G. Zhu, C. Pan, W. Guo, C.Y. Chen, Y. Zhou, R. Yu, Z.L. Wang, Triboelectric-generator-driven pulse electrodeposition for micropatterning. Nano Lett. 12(9), 4960-4965 (2012). doi: $10.1021 / \mathrm{nl} 302560 \mathrm{k}$

75. F.R. Fan, W. Tang, Z.L. Wang, Flexible nanogenerators for energy harvesting and self-powered electronics. Adv. Mater. 28(22), 4283-4305 (2016). doi:10.1002/adma.201504299

76. J. Chen, J. Yang, Z. Li, X. Fan, Y. Zi et al., Networks of triboelectric nanogenerators for harvesting water wave energy: a potential approach toward blue energy. ACS Nano 9(3), 3324-3331 (2015). doi:10.1021/acsnano.5b00534

77. J. Chen, J. Yang, H. Guo, Z. Li, L. Zheng, Y. Su, Z. Wen, X. Fan, Z.L. Wang, Automatic mode transition enabled robust triboelectric nanogenerators. ACS Nano 9(12), 12334-12343 (2015). doi:10.1021/acsnano.5b05618

78. Z. Wen, H. Guo, Y. Zi, M.H. Yeh, X. Wang, J. Deng, J. Wang, S. Li, C. Hu, L. Zhu, Z.L. Wang, Harvesting broad frequency band blue energy by a triboelectric-electromagnetic hybrid nanogenerator. ACS Nano 10(7), 6526-6534 (2016). doi:10. 1021/acsnano.6b03293

79. Y. Zi, H. Guo, Z. Wen, M.H. Yeh, C. Hu, Z.L. Wang, Harvesting low-frequency $(<5 \mathrm{~Hz})$ irregular mechanical energy: a possible killer application of triboelectric nanogenerator. ACS Nano 10(4), 4797-4805 (2016). doi:10.1021/acsnano.6b01569 
80. Q. Liang, X. Yan, Y. Gu, K. Zhang, M. Liang, S. Lu, X. Zheng, Y. Zhang, Highly transparent triboelectric nanogenerator for harvesting water-related energy reinforced by antireflection coating. Sci. Rep. 5, 9080 (2015). doi:10.1038/srep09080

81. Q. Liang, Z. Zhanga, X. Yan, Y. Gu, Y. Zhao, G. Zhang, S. Lu, Q. Liao, Y. Zhang, Functional triboelectric generator as selfpowered vibration sensor with contact mode and non-contact mode. Nano Energy 14, 209-216 (2015). doi:10.1016/j.nanoen. 2014.07.010

82. J. Wang, Z. Wen, Y. Zi, P. Zhou, J. Lin, H. Guo, Y. Xu, Z.L. Wang, All-plastic-materials based self-charging power system composed of triboelectric nanogenerators and supercapacitors. Adv. Funct. Mater. 26(7), 1070-1076 (2016). doi:10.1002/adfm. 201504675

83. X. Wang, Z. Wen, H. Guo, C. Wu, X. He, L. Lin, X. Cao, Z.L. Wang, Fully packaged blue energy harvester by hybridizing a rolling triboelectric nanogenerator and an electromagnetic generator. ACS Nano 10(12), 11369-11376 (2016). doi:10.1021/ acsnano.6b06622

84. Z. Wen, M.H. Yeh, H. Guo, J. Wang, Y. Zi et al., Self-powered textile for wearable electronics by hybridizing fiber-shaped nanogenerators, solar cells, and supercapacitors. Sci. Adv. 2(10), e1600097 (2016). doi:10.1126/sciadv.1600097

85. H. Guo, M.H. Yeh, Y.C. Lai, Y. Zi, C. Wu, Z. Wen, C. Hu, Z.L. Wang, All-in-one shape-adaptive self-charging power package for wearable electronics. ACS Nano 10(11), 10580-10588 (2016). doi:10.1021/acsnano.6b06621

86. Q. Liang, X. Yan, X. Liao, S. Cao, S. Lu, X. Zheng, Y. Zhang, Integrated active sensor system for real time vibration monitoring. Sci. Rep. 5, 16063 (2015). doi:10.1038/srep16063

87. Q. Zhang, Q. Liang, Q. Liao, F. Yi, X. Zheng, M. Ma, F. Gao, Y. Zhang, Service behavior of multifunctional triboelectric nanogenerators. Adv. Mater. (article in press) (2017). doi:10.1002/ adma.201606703

88. Y. Zi, H. Guo, J. Wang, Z. Wen, S. Li, C. Hu, Z.L. Wang, An inductor-free auto-power-management design built-in triboelectric nanogenerators. Nano Energy 31, 302-310 (2017). doi:10.1016/j.nanoen.2016.11.025

89. Y. Zi, S. Niu, J. Wang, Z. Wen, W. Tang, Z.L. Wang, Standards and figure-of-merits for quantifying the performance of triboelectric nanogenerators. Nat. Commun. 6, 8376 (2015). doi:10. 1038/ncomms 9376

90. Y. Zi, J. Wang, S. Wang, S. Li, Z. Wen, H. Guo, Z.L. Wang, Effective energy storage from a triboelectric nanogenerator. Nat. Commun. 7, 10987 (2016). doi:10.1038/ncomms10987

91. H. Zhang, Y. Yang, Y. Su, J. Chen, C. Hu et al., Triboelectric nanogenerator as self-powered active sensors for detecting liquid/gaseous water/ethanol. Nano Energy 2(5), 693-701 (2013). doi:10.1016/j.nanoen.2013.08.004

92. Z.H. Lin, G. Cheng, W. Wu, K.C. Pradel, Z.L. Wang, Dualmode triboelectric nanogenerator for harvesting water energy and as a self-powered ethanol nanosensor. ACS Nano 8(6), 6440-6448 (2014). doi:10.1021/nn501983s

93. S.H. Shin, Y. Kwon, Y.H. Kim, J.Y. Jung, J. Nah, Triboelectric hydrogen gas sensor with pd functionalized surface. Nanomaterials 6(10), 186 (2016). doi:10.3390/nano6100186

94. A.S.M.I. Uddin, G.S. Chung, A self-powered active hydrogen sensor based on a high-performance triboelectric nanogenerator using a wrinkle-micropatterned PDMS film. RSC Adv. 6(67), 63030-63036 (2016). doi:10.1039/C6RA07179A

95. Y.H. Ko, G. Nagaraju, S.H. Lee, J.S. Yu, PDMS-based triboelectric and transparent nanogenerators with $\mathrm{ZnO}$ nanorod arrays. ACS Appl. Mater. Interfaces 6(9), 6631-6637 (2014). doi:10.1021/am5018072
96. J.H. Kim, J. Chun, J.W. Kim, W.J. Choi, J.M. Baik, Self-powered, room-temperature electronic nose based on triboelectrification and heterogeneous catalytic reaction. Adv. Funct. Mater. 25(45), 7049-7055 (2015). doi:10.1002/adfm.201503419

97. A.S.M.I. Uddin, G.S. Chung, A self-powered active hydrogen gas sensor with fast response at room temperature based on triboelectric effect. Sens. Actuators B 231, 601-608 (2016). doi:10.1016/j.snb.2016.03.063

98. X. Xue, Y. Fu, Q. Wang, L. Xing, Y. Zhang, Outputting olfactory bionic electric impulse by PANI/PTFE/PANI sandwich nanostructures and their application as flexible, smelling electronic skin. Adv. Funct. Mater. 26(18), 3128-3138 (2016). doi:10.1002/adfm.201505331

99. A.S.M.I. Uddin, U. Yaqoob, G.S. Chung, Improving the working efficiency of a triboelectric nanogenerator by the semimetallic PEDOT:PSS hole transport layer and its application in self-powered active acetylene gas sensing. ACS Appl. Mater. Interfaces 8(44), 30079-30089 (2016). doi:10.1021/ acsami.6b08002

100. Z.L. Wang, J. Chen, L. Lin, Progress in triboelectric nanogenerators as a new energy technology and self-powered sensors. Energy Environ. Sci. 8(8), 2250-2282 (2015). doi:10.1039/ C5EE01532D

101. Z.L. Wang, Triboelectric nanogenerators as new energy technology and self-powered sensors-principles, problems and perspectives. Faraday Discuss. 176, 447-458 (2014). doi:10. 1039/C4FD00159A

102. Z. Wen, J. Chen, M.H. Yeh, H. Guo, Z. Li, X. Fan, T. Zhang, L. Zhu, Z.L. Wang, Blow-driven triboelectric nanogenerator as an active alcohol breath analyzer. Nano Energy 16, 38-46 (2015). doi:10.1016/j.nanoen.2015.06.006

103. P. Wang, Y. Fu, B. Yu, Y. Zhao, L. Xing, X. Xue, Realizing room-temperature self-powered ethanol sensing of $\mathrm{ZnO}$ nanowire arrays by combining their piezoelectric, photoelectric and gas sensing characteristics. J. Mater. Chem. A 3(7), 3529-3535 (2015). doi:10.1039/C4TA06266C

104. Y. Fu, W. Zang, P. Wang, L. Xing, X. Xue, Y. Zhang, Portable room-temperature self-powered/active $\mathrm{H}_{2}$ sensor driven by human motion through piezoelectric screening effect. Nano Energy 8, 34-43 (2014). doi:10.1016/j.nanoen.2014.05.012

105. Y. Fu, Y. Nie, Y. Zhao, P. Wang, L. Xing, Y. Zhang, X. Xue, Detecting liquefied petroleum gas (LPG) at room temperature using $\mathrm{ZnSnO}_{3} / \mathrm{ZnO}$ nanowire piezo-nanogenerator as self-powered gas sensor. ACS Appl. Mater. Interfaces 7(19), 10482-10490 (2015). doi:10.1021/acsami.5b01822

106. D. Zhu, Y. Fu, W. Zang, Y. Zhao, L. Xing, X. Xue, Roomtemperature self-powered ethanol sensor based on the piezosurface coupling effect of heterostructured $\alpha-\mathrm{Fe}_{2} \mathrm{O}_{3} / \mathrm{ZnO}$ nanowires. Mater. Lett. 166, 288-291 (2016). doi:10.1016/j.matlet. 2015.12.106

107. Z. Qu, Y. Fu, B. Yu, P. Deng, L. Xing, X. Xue, High and fast $\mathrm{H}_{2} \mathrm{~S}$ response of $\mathrm{NiO} / \mathrm{ZnO}$ nanowire nanogenerator as a selfpowered gas sensor. Sens. Actuators B 222, 78-86 (2016). doi:10.1016/j.snb.2015.08.058

108. W. Zang, Y. Nie, D. Zhu, P. Deng, L. Xing, X. Xue, Core-shell $\mathrm{In}_{2} \mathrm{O}_{3} / \mathrm{ZnO}$ nanoarray nanogenerator as a self-powered active gas sensor with high $\mathrm{h}_{2}$ s sensitivity and selectivity at room temperature. J. Phys. Chem. C 118(17), 9209-9216 (2014). doi:10.1021/jp500516t

109. D. Zhu, Y. Fu, W. Zang, Y. Zhao, L. Xing, X. Xue, Piezo/active humidity sensing of $\mathrm{CeO}_{2} / \mathrm{ZnO}$ and $\mathrm{SnO}_{2} / \mathrm{ZnO}$ nanoarray nanogenerators with high response and large detecting range. Sens. Actuators B 205, 12-19 (2014). doi:10.1016/j.snb.2014. 08.060 